\title{
A Flicker Noise/IM3 Cancellation Technique for Active Mixer Using Negative Impedance
}

Wei Cheng, Anne Johan Annema, Member, IEEE, Gerard J.M. Wienk and Bram Nauta, Fellow, IEEE

University of Twente, CTIT Institute, IC Design group, Enschede, The Netherlands

Contact Information:

Name: Wei Cheng, Room 2009, Building Carre, Hallenweg 15, University of Twente, 7522NH Enschede, The Netherlands;Phone: +31 53489 2727, Fax: +31 53489 1034, E-mail:w.cheng@utwente.nl

Abstract - This paper presents an approach to simultaneously cancel flicker noise and IM3 in Gilbert-type mixers, utilizing negative impedances. For proof of concept, two prototype double-balanced mixers in $0.16 \mu \mathrm{m}$ CMOS are fabricated. The first demonstration mixer chip was optimized for full IM3 cancellation and partial flicker noise cancellation; this chip achieves 9dB flicker noise suppression, improvements of $10 \mathrm{~dB}$ for IIP $3,5 \mathrm{~dB}$ for conversion gain, and $1 \mathrm{~dB}$ for input $\mathrm{P}_{1 \mathrm{~dB}}$ while the thermal noise increased by $0.1 \mathrm{~dB}$. The negative impedance increases the power consumption for the mixer by $16 \%$, and increases the die area by $8 \%\left(46 \times 28 \mu \mathrm{m}^{2}\right)$. A second demonstration mixer chip aims at full flicker noise cancellation and partial IM3 cancellation, while operating on a low supply voltage $\left(0.67 \times \mathrm{V}_{\mathrm{DD}}\right)$; in this chip, the negative impedance increases the power consumption by $7.3 \%$, and increases the die area by $7 \%\left(50 \times 20 \mu \mathrm{m}^{2}\right)$. For one chip sample, measurements show $>10 \mathrm{~dB}$ flicker noise suppression within $\pm 200 \%$ variation of the negative impedance bias current; for ten randomly selected chip samples $>11 \mathrm{~dB}$ flicker noise suppression is measured.

Index Terms - Active mixer, flicker noise, direct conversion, CMOS, narrowband, receiver, IIP3, linearity, IM3, distortion cancellation, noise cancellation.

\section{INTRODUCTION}

CMOS active mixers have high gain but also suffer from high flicker noise as well as from low linearity. While high flicker noise causes serious sensitivity degradation especially in narrowband 
direct-conversion receivers, mixers with poor linearity limit the dynamic range of the receiver.

Three major techniques have been presented for flicker noise reduction in CMOS active mixers:

1. Dynamic current injection [1-2]: a pMOS cross-coupled pair injects current into the NMOS mixer transconductor stage only at the switching on/off instants (at $0.5 \mathrm{~T}_{\mathrm{LO}}$ ) in such a way that no DC current flows through the switches then. This is reported to suppress the flicker noise leakage from the switching pair.

2. Double LO switch pairs [3]: extra switches in series driven at 2LO frequency are used in such a way that during the switching period little DC current flows through the major switches that are driven by LO signal, thereby reducing flicker noise leakage.

3. RF leakageless static current bleeding with two resonating inductors [4]. Two inductors are connected between the mixer transconductor stage and the current bleeding circuit. The inductors resonate out the tail capacitance and reduce the RF signal leakage to the current bleeding circuit.

In Technique 1, a large LO swing and large headroom is required, increasing the LO power and decreasing the conversion gain due to the use of small $R_{\text {load }}[1]$. Technique 2 needs a stack of three transistors plus the load, which is not suitable for deep-submicrometer technologies with low supply voltages. Technique 3 needs two inductors which consume significant die area. Common to all the techniques in [1-4] is that the effect of the transistor output resistance on the flicker noise leakage is neglected.

In technologies with long-channel transistors where the output capacitance of transistors is dominant in the transistors' output impedance at RF frequencies, the effect of output resistance on flicker noise leakage can be neglected [1-4]. However, nowadays CMOS technologies offer 
transistors with $f_{\mathrm{T}}$ well above $100 \mathrm{GHz}$, at the same time with lower transistor output resistance and lower supply voltage [5]. Neglecting the effect of output resistance in deep-submicrometer technologies can yield a significant underestimation of the output flicker noise [7]. Taking into account the effect of both output resistance and output capacitance on flicker noise leakage, in this paper we propose a combined flicker noise/IM3 cancellation technique that uses a negative impedance to minimize the flicker noise leakage from the switching pair and to simultaneously improve the linearity. Section II presents the circuit theory behind this flicker noise/IM3 cancellation technique. Section III and IV show a circuit implementation and the measurement and simulation results; the results are summarized in section $\mathrm{V}$.

\section{FLICKER NOISE/IM3 CANCELLATION USING A NEGATIVE IMPEDANCE}

\section{A. Flicker noise leakage in Gilbert mixers}

The double-balanced Gilbert mixer shown in Fig. 1a is widely used as the active downconverter in CMOS receivers. Transistor $\mathrm{M}_{3 \mathrm{a}} / \mathrm{M}_{3 \mathrm{~b}}$ convert $\mathrm{v}_{\text {in }}$ into current that is commuting via switch pair $\mathrm{M}_{1 \mathrm{a}} / \mathrm{M}_{2 \mathrm{a}}$ and $\mathrm{M}_{1 \mathrm{~b}} / \mathrm{M}_{2 \mathrm{~b}}$ respectively. The flicker noise output of the Gilbert mixer, $v_{f l, o u t}(t)$, is dominated by the switch pair $M_{1} / M_{2}$, while transistor $M_{3}$ is causing thermal noise folding [6-7]. Assuming perfect symmetry in the mixer, the flicker noise leakage mechanism from each switch is the same: it is hence sufficient to focus on flicker noise leakage from one of the switch pair transistors. In [7] the time-varying small signal model shown in Fig. $1 \mathrm{~b}$ is used to analyze the flicker noise contributed by $\mathrm{M}_{1 \mathrm{a}}$ in one LO period at the mixer output $\left(v_{f l, o u t}^{M_{1 a}}(t)\right)$. The flicker noise of $\mathrm{M}_{1 \mathrm{a}}$ is modeled by the equivalent gate-referred root mean square (rms) noise voltage $v_{f l, i n}^{M_{1} a}$. For a first order approximation of $v_{f l, o u t}^{M_{1 a}}$, a few assumptions are made:

- The LO signal is modeled by a trapezoid shown in Fig. 2a with a rise/fall time (e.g. $\left.\mathrm{t}_{3}-\mathrm{t}_{2}\right)$ 
equal to $\alpha \cdot T_{L O}$.

- In $\left(t_{1}, t_{2}\right), M_{2 a}$ and $M_{2 b}$ are off while in $\left(t_{3}, t_{4}\right) M_{1 a}$ and $M_{1 b}$ are off.

Taking into account the transconductance of $\mathrm{M}_{1}, \mathrm{M}_{2}$ and $\mathrm{M}_{3}$, and the output admittance of $\mathrm{M}_{3}\left(Y_{d s}^{M_{3} a}=\right.$ $\left.g_{d s}^{M_{3 a}}+j \omega_{L O} C_{d s}^{M_{3 a} a}\right)$, the flicker noise contribution of $\mathrm{M}_{1 \mathrm{a}}$ at $\mathrm{t}_{1}, \frac{T_{L O}}{2}$ and $\mathrm{t}_{3}$ in Fig. 2a are given by:

$$
\begin{aligned}
& \left.v_{f l, \text { out }}^{M_{1 a}}\right|_{t_{1}}=\left.\frac{-g_{m}^{M_{1} R_{L} R_{d s}}}{g_{m}^{M_{1} a}+Y_{d s}^{M_{3} a}} v_{f l, \text { in }}^{M_{1} a}\right|_{t_{1}} \\
& \left.v_{f l, \text { out }}^{M_{1 a}}\right|_{\frac{T_{L O}}{2}}=-\left.g_{m}^{M_{1 a} a} R_{L} v_{f l, \text { in }}^{M_{1 a} a}\right|_{\frac{T_{L O}}{2}} \\
& \left.v_{f l, o u t}^{M_{1 a} a}\right|_{t_{3}}=0
\end{aligned}
$$

In ( $\left.t_{1}, t_{2}\right) M_{1 a}$ and $M_{3 a}$ form a cascode amplifier. Due to the finite output impedance of $M_{3 a}$ in deepsubmicrometer CMOS, the noise contribution from the cascode transistor $\mathrm{M}_{1}$, given by (1), cannot be neglected. In $\left(\mathrm{t}_{2}, \mathrm{t}_{3}\right)$ both $\mathrm{M}_{1 \mathrm{a}}$ and $\mathrm{M}_{2 \mathrm{a}}$ are on, while at $\frac{T_{L O}}{2} \mathrm{M}_{1 \mathrm{a}}$ and $\mathrm{M}_{2 \mathrm{a}}$ act as a balanced differential pair. In this period of time, the output impedance of $\mathrm{M}_{3}$ has a negligibly small effect on $v_{f l, o u t}^{M_{1 a}}$ as shown by (2). In ( $\left.\mathrm{t}_{3}, \mathrm{t}_{4}\right) \mathrm{M}_{1 \mathrm{a}}$ is assumed to be off, thus $v_{f l, o u t}^{M_{1 a}}$ is zero. Note that the integral (or area) of $v_{f l, o u t}^{M_{1 a}}(t)$ shown in Fig. 2(b) and (c) corresponds to the flicker noise leakage that involves no frequency translation [7]. As a result, the flicker noise at the mixer output contributed by $\mathrm{M}_{1 \mathrm{a}} / \mathrm{M}_{2 \mathrm{a}}$ and $\mathrm{M}_{1 \mathrm{~b}} / \mathrm{M}_{2 \mathrm{~b}}$ is

$$
\begin{aligned}
& S_{f l, \text { out }}=4 \times\left|\int_{0}^{T_{L O}} \operatorname{Re}\left[v_{f l, \text { out }}^{M_{1 a}}(t)\right] d t+j \times \int_{0}^{T_{L O}} \operatorname{Im}\left[v_{f l, \text { out }}^{M_{1 a}}(t)\right] d t\right|^{2} \\
& =\frac{4}{T_{L O}{ }^{2}} \times\left|\left(t_{2}-t_{1}\right) \cdot v_{f l, \text { out }}^{M_{1 a}}\right|_{t_{1}}+t_{1} \cdot\left(\left.v_{f l, \text { out }}^{M_{1 a}}\right|_{\frac{T_{L O}}{2}}-\left.v_{f l, \text { out }}^{M_{1 a}}\right|_{t_{1}}\right)+\left.\left.\left(t_{3}-\frac{T_{L O}}{2}\right) \cdot v_{f l, \text { out }}^{M_{1}}\right|_{\frac{T_{L O}}{2}}\right|^{2}
\end{aligned}
$$

For the symmetrical LO signal shown in Fig. $2 \mathrm{a}$, with a rise/fall time equal to $\alpha \cdot T_{L O}$, the time instants $t_{1}, t_{2}$ and $t_{3}$ can be rewritten as $t_{1}=\alpha T_{L O} / 2, t_{2}=(1-\alpha) T_{L O} / 2, t_{3}=(1+\alpha) T_{L O} / 2$. This enables rewriting (4) into 


$$
S_{f l, \text { out }}=\left|(1-3 \alpha) \cdot v_{f l, \text { out }}^{M_{1 a}}\right|_{t_{1}}+\left.\left.2 \alpha v_{f l, \text { out }}^{M_{1 a}}\right|_{\frac{T_{L O}}{2}}\right|^{2}
$$

\section{B. Negative impedance for flicker noise cancellation}

To minimize the integral of $v_{f l, o u t}^{M_{1} a}(t)$ in (4) --- hence to minimize the flicker noise leakage --- we apply a negative impedance $Y_{n e g}=G_{n e g}+j \omega C_{n e g}$ between the drain of $\mathrm{M}_{3 \mathrm{a}}$ and $\mathrm{M}_{3 \mathrm{~b}}$ as shown in Fig. 3. Using the model shown in Fig.3b, this yields a different $v_{f l, o u t}^{M_{1 a}}$ for the time interval $\left(\mathrm{t}_{1}-\mathrm{t}_{2}\right)$ :

$$
\begin{aligned}
& \left.v_{f l, \text { out }}^{M_{1 a}}\right|_{t_{1}}=\left.\frac{-g_{m}^{M_{1} R_{L}}\left(Y_{d s}^{M_{3} a}+2 Y_{\text {neg }}\right) v_{f l, \text { in }}^{M_{1 a} a}}{g_{m}^{M_{1} a}+Y_{d s}^{M_{3} a}+2 Y_{\text {neg }}}\right|_{t_{1}} \\
& v_{f l, \text { out }}^{\left.M_{1 a}\right|_{\frac{T_{L O}}{2}}}=-g_{m}^{\left.M_{1 a} R_{L} v_{f l, \text { in }}^{M_{1 a}}\right|_{\frac{T_{L O}}{2}}} \\
& \left.v_{f l, \text { out }}^{M_{1 a}}\right|_{t_{3}}=0
\end{aligned}
$$

Eq. (6) shows that for $C_{n e g} \approx-0.5 C_{\text {tail }}$ and $G_{n e g} \in\left[\frac{-g_{d s}^{M_{3} a_{2}}-g_{m}^{M_{1} a}}{2}, \frac{-g_{d s}^{M_{3 a}}}{2}\right]$ the sign of the real part of $\left.v_{f l, o u t}^{M_{1 a}}\right|_{t_{1}}$ changes from negative to positive (a detailed derivation is presented in the appendix). At $\frac{T_{L O}}{2}$ the negative impedance has no effect on $v_{f l, o u t}^{M_{1 a}}$ as shown in (7) since $\mathrm{M}_{1 \mathrm{a}}$ and $\mathrm{M}_{2 \mathrm{a}}$ act as a balanced differential pair. In $\left(\mathrm{t}_{3}, \mathrm{t}_{4}\right) \mathrm{M}_{1 \mathrm{a}}$ is off, thus $v_{f l, o u t}^{M_{1 a}}$ is zero. Now the new approximated waveform of $v_{f l, o u t}^{M_{1 a}}(t)$ is shown in Fig. $4 \mathrm{~b}$. The negative impedance $Y_{\text {neg }}$ changes the real part of $v_{f l, o u t}^{M_{1 a}}$ in $\left(\mathrm{t}_{1}, \mathrm{t}_{2}\right)$ from negative to positive value, which enables minimization of the area of $v_{f l, o u t}^{M_{1 a}}(t)$ in one LO period. This leads to the cancellation of the flicker noise leakage from the switching pair $\left(\mathrm{M}_{1 \mathrm{a}} / \mathrm{M}_{2 \mathrm{a}}\right.$ and $\left.\mathrm{M}_{1 \mathrm{~b}} / \mathrm{M}_{2 \mathrm{~b}}\right)$. For a complete flicker noise leakage cancellation, (5) equates to zero. Together with (6-7), this equation gives the condition for complete flicker noise leakage cancellation:

$$
\left\{\begin{array}{c}
G_{n e g}=-\frac{g_{d s}^{M_{3} a}}{2}-\frac{\alpha g_{m}^{M_{1} a}}{(1-\alpha)} \\
C_{n e g}=-\frac{C_{\text {tail }}}{2}
\end{array}\right.
$$

Note that for complete cancellation across process and temperature spread, $G_{\text {neg }}$ should track the variation of the sum of $g_{d s}$ and $g_{m}$ and $C_{n e g}$ should track $C_{\text {tail }}$. 


\section{Negative-impedance impact on gain and thermal noise}

It was derived in [7] that the first-order Fourier coefficient of the instantaneous voltage gain $F^{M_{3}}(t)=v_{\text {out }} / v_{\text {in }}$ in one LO period corresponds to the conversion gain of a mixer. Using the model shown in Fig. 5a, a sufficiently accurate approximation of $F^{M_{3}}(t)$ is given in Fig. 5b with

$$
\begin{aligned}
& \left.F^{M_{3}}\right|_{t_{1}}=\left.\frac{-g_{m}^{M_{1} a} g_{m}^{M_{3} a_{L}}}{g_{m}^{M_{1} a}+Y_{d s}^{M_{3} a}+2 Y_{n e g}}\right|_{t_{1}} \\
& \left.F^{M_{3}}\right|_{\frac{T_{L O}}{2}}=0 \\
& \left.F^{M_{3}}\right|_{t_{3}}=-\left.F^{M_{3}}\right|_{t_{1}}
\end{aligned}
$$

At $\left(t_{1}, t_{2}\right), M_{2 a} / M_{2 b}$ are off and $M_{1 a} / M_{3 a}$ and $M_{1 b} / M_{3 b}$ (see Fig. 3(a)) forms a differential cascode common-source amplifier. At $\left(t_{3}, t_{4}\right) M_{1 a} / M_{1 b}$ are off and $M_{2 a} / M_{3 a}$ and $M_{2 b} / M_{3 b}$ form a differential cascode common-source amplifier. At $\frac{T_{L O}}{2} \mathrm{M}_{1 \mathrm{a}} / \mathrm{M}_{2 \mathrm{a}}$ and $\mathrm{M}_{1 \mathrm{~b}} / \mathrm{M}_{2 \mathrm{~b}}$ are on and there is no output due to the differential symmetry. This yields the voltage conversion gain

$$
V_{\text {gain }}=\left.\frac{2 \sin (\alpha \pi)}{\pi^{2} \alpha} F^{M_{3}}\right|_{t_{1}}=\left.\frac{2 \sin (\alpha \pi)}{\pi^{2} \alpha} \frac{-g_{m}^{M_{1} a} g_{m}^{M_{3} a} R_{L}}{g_{m}^{M_{1} a}+Y_{d s}^{M_{3} a}+2 Y_{\text {neg }}}\right|_{t_{1}}
$$

Equation (13) shows that the conversion gain is increased under partial flicker noise cancelling condition $\left(G_{n e g} \in\left[\frac{-g_{d s}^{M_{3} a}-g_{m}^{M_{1 a} a}}{2}, \frac{-g_{d s}^{M_{3 a}}}{2}\right]\right.$ and $\left.C_{n e g} \approx-0.5 C_{\text {tail }}\right)$. The reason for this increase is that $\mathrm{G}_{\text {neg }}$ increases the output impedance for the gm stage and as a result more RF signal current flows to the load $\mathrm{R}_{\text {load. }}$.

Fig. 6 shows the noise model for the mixer, where two non-correlated noise current $i_{\text {tha }}^{Y_{\text {neg }}}$ and $i_{\text {thb }}^{Y_{\text {neg }}}$ model the noise of $\mathrm{Y}_{\text {neg. }}$. Note that the noise of the negative impedance contributes to the mixer output by the same transfer function as the noise of $\mathrm{M}_{3 \mathrm{a}} / \mathrm{M}_{3 \mathrm{~b}}$. Therefore, $\mathrm{Y}_{\text {neg }}$ only contributes to thermal noise while no flicker noise leaks to the output for a symmetric mixer. As a result, the mixer thermal noise is dominantly contributed by the thermal-noise folding of: $M_{3 a} / M_{3 b}, M_{1 a} / M_{1 b}, M_{2 a} / M_{2 b}$, 
the load $R_{\text {Load }}$, the input source impedance $R_{s}$ and the negative impedance $Y_{\text {neg. }}$. Assuming perfect input matching, the single-side band noise figure (NF) for high IF (thermal noise dominated) is then given by

$$
\begin{aligned}
& N F_{S S B} \approx \frac{2 S_{M 3 a, \text { out }}+4 S_{M 1 \text { a,out }}+S_{R s, \text { out }}+S_{\text {Yneg }}+S_{\text {Rload }, \text { out }}}{0.5 S_{R s, \text { out }}} \\
& =2 \frac{\left.\left[\left(\frac{i_{\text {th } 3 a}}{g_{m 3}}\right)^{2}+4 \times\left(\frac{\left(g_{d s}^{M_{3 a}}+2 G_{\text {neg }}\right) i_{\text {th } 1 a}}{g_{m 1} g_{m 3}}\right)^{2}+k T R_{S}+\left(\frac{i_{\text {th }}^{Y_{\text {neg }}}}{g_{m 3}}\right)^{2}\right]\right|_{t_{1}}+\frac{4 k T R_{\text {load }}}{\left.\left(1-\frac{4 \alpha}{3}\right)\left|F^{M_{3}}\right|_{t_{1}}\right|^{2}}}{k T R_{S}}
\end{aligned}
$$

where the five terms respectively account for the thermal noise from the transconductor stage $\mathrm{M}_{3 \mathrm{a}} / \mathrm{M}_{3 \mathrm{~b}}$, the thermal noise from the switching stage $\left(\mathrm{M}_{1 \mathrm{a}} / \mathrm{M}_{1 \mathrm{~b}}\right.$ and $\left.\mathrm{M}_{2 \mathrm{a}} / \mathrm{M}_{2 \mathrm{~b}}\right)$, the thermal noise from the input source impedance $\mathrm{R}_{\mathrm{s}}$, the thermal noise from the negative impedance and the thermal noise from the load. Although the extra noise by $\mathrm{Y}_{\text {neg }}$ increases the thermal noise NF, due to the increased conversion gain (larger $\left.F^{M_{3}}\right|_{t_{1}}$ ), the input referred noise due to the load $\mathrm{R}_{\mathrm{Load}}$ is decreased. As a result, the thermal noise increase due to $Y_{\text {neg }}$ can be small.

\section{Negative impedance for IM3 distortion cancellation}

It is shown in [7] that the IM3 of the time-varying mixer can be estimated by one time-invariant IM3 calculation at the maximum of the LO signal. The circuit model shown in Fig. 7 is now used to demonstrate the concept of using negative impedance for IM3 cancellation. When the LO signal reaches its positive maximum at $t_{1}, M_{1 a} / M_{1 b}$ are fully on and $M_{2 a} / M_{2 b}$ are fully off. The IM3 distortion current $i_{I M 3}^{M_{1 a}}, i_{I M 3}^{M_{1 b}}, i_{I M 3}^{M_{3 a}}$ and $i_{I M 3}^{M_{3 b}}$ are generated by the voltage swing across the transistor terminals. Given the differential circuit topology we can assume $i_{I M 3}^{M_{1 a}}=-i_{I M 3}^{M_{1} b}=i_{I M 3}^{M_{1}}$ and $i_{I M 3}^{M_{3} a}=-i_{I M 3}^{M_{3 b}}=i_{I M 3}^{M_{3}}$. For a first-order approximation, only the transconductance of $\mathrm{M}_{1}, \mathrm{M}_{2}$ and $\mathrm{M}_{3}$, and the output admittance of $\mathrm{M}_{3}$ are taken into account, which yields

$$
v_{\text {out }}^{I M 3}=\frac{-2 g_{m}^{M_{1}} R_{L}}{g_{m}^{M_{1}}+Y_{d s}^{M_{3}}+2 Y_{n e g}} i_{I M 3}^{M_{3}}+\frac{-2\left(Y_{d s}^{M_{3}}+2 Y_{n e g}\right) R_{L}}{g_{m}^{M_{1}}+Y_{d s}^{M_{3}}+2 Y_{\text {neg }}} i_{I M 3}^{M_{1}}
$$




$$
\begin{aligned}
& i_{I M 3}^{M_{3}}=f_{I M 3}^{M_{3}}\left(v_{g s}^{M_{3} a}, v_{d s}^{M_{3} a}\right)=f_{I M 3}^{M_{3}}\left[\frac{v_{\text {in }}}{2}, \frac{-g_{m}^{M_{3}} v_{\text {in }}}{2\left(g_{m}^{M_{1}}+Y_{d s}^{M_{3}}+2 Y_{n e g}\right)}\right] \\
& i_{I M 3}^{M_{1}}=f_{I M 3}^{M_{1}}\left(v_{g s}^{M_{1} a}, v_{d s}^{M_{1 a}}\right)=f_{I M 3}^{M_{1}}\left[\frac{g_{m}^{M_{3}} v_{i n}}{2\left(g_{m}^{M_{1}}+Y_{d s}^{M_{3}}+2 Y_{n e g}\right)}, \frac{-g_{m}^{M_{3}}\left(-1+g_{m}^{M_{1}} R_{L}\right) v_{i n}}{2\left(g_{m}^{M_{1}}+Y_{d s}^{M_{3}}+2 Y_{n e g}\right)}\right]
\end{aligned}
$$

Equation (16) and (17) describe the fact that via transistor nonlinearity (denoted by the function $f_{d}$ ) the distortion current of a transistor is due to both the voltage swing across its gate-source and to its drain-source voltage swing (assuming the distortion related to the bulk-source voltage swing is insignificant).

For $G_{n e g} \in\left[\frac{-g_{d s}^{M_{3} a}-g_{m}^{M_{1 a}}}{2}, \frac{-g_{d s}^{M_{3} a}}{2}\right]$ and $C_{n e g} \approx-0.5 C_{\text {tail }}$,

- $\quad$ Eq. (16-17) show that the gate-source and drain-source voltage swing respectively for $\mathrm{M}_{1 \mathrm{a}}$ and $\mathrm{M}_{3 \mathrm{a}}$ have the same polarity. Thus $i_{I M 3}^{M_{3}}$ and $i_{I M 3}^{M_{1}}$ have the same polarity, given that both $\mathrm{M}_{1 \mathrm{a}}$ and $\mathrm{M}_{3 \mathrm{a}}$ are biased in the saturation region.

- Eq. (15) shows that the gain factor for distortion currents $i_{I M 3}^{M_{3}}$ and $i_{I M 3}^{M_{1}}$ have opposite signs. This enables cancellation of the distortion contributions caused by $i_{I M 3}^{M_{3}}$ and $i_{I M 3}^{M_{1}}$.

Equating (15) to zero gives that for a complete IM3 cancellation

$$
\left\{\begin{array}{c}
G_{n e g}=-\frac{g_{d s}^{M_{3}}}{2}-\frac{g_{m}^{M_{1}} i_{d}^{M_{3}}}{2 i_{d}^{M_{1}}} \\
C_{n e g}=-\frac{C_{\text {tail }}}{2}
\end{array}\right.
$$

Under partial IM3 cancelling condition $\left(G_{n e g} \in\left[\frac{-g_{d s}^{M_{3} a}-g_{m}^{M_{1 a}}}{2}, \frac{-g_{d s}^{M_{3} a}}{2}\right]\right.$ and $\left.C_{n e g} \approx-0.5 C_{\text {tail }}\right)$, the distortion current polarity of each transistor within the mixer remains unchanged. For the switching stage $\left(\mathrm{M}_{1 \mathrm{a}} / \mathrm{M}_{1 \mathrm{~b}}\right.$ and $\left.\mathrm{M}_{2 \mathrm{a}} / \mathrm{M}_{2 \mathrm{~b}}\right)$, the negative impedance changes the amplifying factor of their distortion current from negative to positive. This enables the IM3 cancellation between the transconductor stage $\left(\mathrm{M}_{3 \mathrm{a}} / \mathrm{M}_{3 \mathrm{~b}}\right)$ and the switching stage $\left(\mathrm{M}_{1 \mathrm{a}} / \mathrm{M}_{1 \mathrm{~b}}\right.$ and $\left.\mathrm{M}_{2 \mathrm{a}} / \mathrm{M}_{2 \mathrm{~b}}\right)$. As the IM3 cancellation depends on the scaling and subtraction of distortion currents of the switching stage and $g_{m}$ stage, we use Monte Carlo simulations to evaluate its sensitivity over device mismatches and process spread; the results are 
shown in section IV. A.

\section{E. Simulation verification}

To illustrate the validity of the proposed theory of flicker noise and IM3 cancellation, in this section we show some simulation results for the mixer shown in Fig. 3a using an ideal negative resistance and an ideal negative capacitance in parallel to implement $Y_{\text {neg. }}$. The bias and dimension condition for this mixer is the same as one (MixerD) that will be discussed in section III. In simulations, for $0.9 \mathrm{GHz} \mathrm{LO}$, we sweep the negative resistance while using a capacitance of $-80 \mathrm{fF}$.

Both simulated and calculated (using (5)-(8)) DSB NF in Fig. 8 (a) clearly show that either complete or partial cancellation of flicker noise can be achieved by using an ideal $\mathrm{Y}_{\text {neg }}$. The sweet spot for complete flicker noise cancellation is by nature sensitive to device mismatch and PVT variations, illustrated by the notch around the optimum $\mathrm{Y}_{\text {neg }}=-950$. Partial flicker noise cancellation is less sensitive to device mismatch and PVT variations: for this example, within $\pm 15 \%$ variation of $Y_{\text {neg }}$ at the NF notch more than $20 \mathrm{~dB}$ flicker cancellation is achieved. Due to some circuit analysis simplification in deriving (9), the optimal $\mathrm{Y}_{\text {neg }}$ for complete flicker noise according to (9) is somewhat different than actual (following from simulations) value. Fig. 8 (b) shows similar results for distortion cancellation, illustrating that IM3 distortion cancellation can be achieved using a properly designed $\mathrm{Y}_{\text {neg }}$ and illustrating that (15)-(18) provide a good prediction for the actual (simulated) IM3 distortion cancellation.

\section{F. Summary}

It can be concluded that a negative impedance $\left(G_{n e g} \in\left[\frac{-g_{d s}^{M_{3} a_{-}}-g_{m}^{M_{1 a}}}{2}, \frac{-g_{d s}^{M_{3} a}}{2}\right]\right.$ and $C_{n e g} \approx$ $\left.-0.5 C_{\text {tail }}\right)$ reduces the flicker noise leakage from the switching stage $\left(\mathrm{M}_{1 \mathrm{a}} / \mathrm{M}_{1 \mathrm{~b}}\right.$ and $\left.\mathrm{M}_{2 \mathrm{a}} / \mathrm{M}_{2 \mathrm{~b}}\right)$ by averaging out the flicker noise leakage transfer function. Note that this is very similar to flicker noise 
suppression in chopper amplifiers [8]. Using a negative impedance, also the conversion gain is increased while the thermal noise may be slightly degraded. For a perfect symmetric mixer no flicker noise will be introduced by the negative impedance.

Using negative impedance in the specified range also enables partial IM3 cancellation between the transconductor stage $\left(\mathrm{M}_{3 \mathrm{a}} / \mathrm{M}_{3 \mathrm{~b}}\right)$ and the switching stage $\left(\mathrm{M}_{1 \mathrm{a}} / \mathrm{M}_{1 \mathrm{~b}}\right.$ and $\left.\mathrm{M}_{2 \mathrm{a}} / \mathrm{M}_{2 \mathrm{~b}}\right)$. The exact optimum of $\mathrm{Y}_{\text {neg }}$ is in general a little different for complete flicker noise cancellation and complete IM3 cancellation. Hence $Y_{\text {neg }}$ can be designed for either full flicker noise/partial IM3 cancellation or full IM3/partial flicker noise cancellation.

\section{CIRCUIT IMPLEMENTATION}

\section{A. Circuit implementation of the negative impedance}

To prove this flicker noise/IM3 cancellation concept, the circuit shown in Fig. 9 is implemented in a standard $0.16 \mu \mathrm{m}$ CMOS process. The negative impedance is implemented by the cross-coupled pair $\mathrm{M}_{4 \mathrm{a}} / \mathrm{M}_{4 \mathrm{~b}}$ with source degeneration provided by capacitor $\left(C_{S}\right)$ and current source $\mathrm{M}_{5 \mathrm{a}} / \mathrm{M}_{5 \mathrm{~b}}$ [9-10]. The pMOS-based negative impedance enables dc current reuse of the negative impedance by the mixer's transconductor stage. As a first-order estimation, the value of the negative impedance --- taking only the transconductance of $\mathrm{M}_{4}$ and $\mathrm{M}_{5}$ and the output impedance of $\mathrm{M}_{5}$ into account --- is:

$$
\left\{\begin{array}{c}
G_{n e g}=-\frac{g_{m}^{M_{4}}\left[g_{d s}^{M_{5}}\left(g_{d s}^{M_{5}}+g_{m}^{M_{4}}\right)+\left(c_{d s}^{M_{5}}+2 C_{s}\right)^{2} \omega^{2}\right]}{2\left[\left(g_{d s}^{M_{5}}+g_{m}^{M_{4}}\right)^{2}+\left(C_{d s}^{M_{5}}+2 C_{s}\right)^{2} \omega^{2}\right]} \\
C_{n e g}=-\frac{g_{m}^{M_{4}}\left(c_{d s}^{M_{5}}+2 C_{s}\right)^{2} \omega}{2\left[\left(g_{d s}^{M_{5}}+g_{m}^{M_{4}}\right)^{2}+\left(c_{d s}^{M_{5}}+2 C_{s}\right)^{2} \omega^{2}\right]}
\end{array}\right.
$$

Assuming $g_{m}^{M_{4}} \gg g_{d s}^{M_{5}}$, and denoting $\frac{g_{m}^{M_{4}}}{c_{d s}^{M_{5}}+2 C_{s}}=\omega_{t}$, then for $\omega \ll \omega_{t}$ (19) reduces to 


$$
\left\{\begin{array}{c}
G_{n e g} \approx-\frac{g_{m}^{M_{4}}\left(\frac{\omega}{\omega_{t}}\right)^{2}}{2\left[1+\left(\frac{\omega}{\omega_{t}}\right)^{2}\right]} \approx-\frac{g_{m}^{M_{4}}}{2} \\
C_{n e g} \approx-\frac{C_{d s}^{M_{5}+2 C_{s}}}{2\left[1+\left(\frac{\omega}{\omega_{t}}\right)^{2}\right]} \approx-\frac{C_{d s}^{M_{5}+2 C_{s}}}{2}
\end{array}\right.
$$

Equation (20) shows that the $Y_{\text {neg }}$ for either full flicker noise cancellation or full IM3 cancellation can be obtained by setting a suitable value both for the transconductance of $\mathrm{M}_{4}$ and for the degeneration capacitance $C_{s}$. For minimal chip area, two anti-parallel poly-diffusion capacitors are used for $C_{S}$ instead of a fringe capacitor.

Equation (20) also shows that the implemented $Y_{\text {neg }}$ is frequency dependent: the negative conductance $\left(\mathrm{G}_{\mathrm{neg}}\right)$ has a high-pass characteristic while the negative capacitance $\left(\mathrm{C}_{\text {neg }}\right)$ presents a

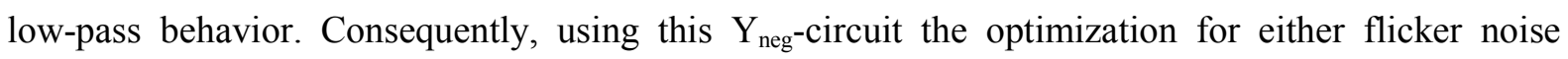
cancellation or for IM3 cancellation is frequency-dependent. In order to demonstrate the effect of the frequency-dependency of the $\mathrm{Y}_{\text {neg }}$ circuit, Fig. 10 shows simulation results for one of the designed mixer circuits in section III: MixerD, which is designed for full IM3 cancellation and partial flicker noise cancellation at $0.9 \mathrm{GHz}$. Design details of MixerD is shown in section III. B.

Fig. 10 (a) shows IIP3 as a function of the LO frequency, swept from $0.1 \mathrm{GHz}$ to $2 \mathrm{GHz}$. The IIP3 peak around $0.9 \mathrm{GHz}$ shows that $\mathrm{Y}_{\text {neg }}$ circuit is optimized for this frequency. For higher frequencies, the IM3 cancellation degrades, mainly due to the less negative capacitance provided by the $Y_{\text {neg }}$ circuit with increasing frequency. As a result, the phase difference between the distortion currents of the switching stage and of the gm stage then deviates from 180 degrees. For LO frequencies lower than $0.8 \mathrm{GHz}$, the distortion cancellation remain effective since parasitics has less effect. Fig. 10 (b) shows that the $Y_{\text {neg }}$ circuit achieves a complete flicker noise cancellation at around $0.2 \mathrm{GHz}$ and that only partial flicker noise cancellation is achieved at higher frequencies. 
In summary, the $G_{\text {neg }}$ and $C_{\text {neg }}$ provided by our $Y_{\text {neg }}$ circuit is frequency-dependent. As a result, a complete IM3 distortion or a complete flicker noise cancellation provided by the circuit implementation shown in Fig. 8 is narrowband. Tuning the bias of the $\mathrm{Y}_{\text {neg }}$ circuit and using tunable capacitors for Cs, a tunable negative impedance can be provided by the $\mathrm{Y}_{\text {neg }}$ circuit for various frequencies which may enable complete IM3 distortion or complete flicker noise cancellation for multiband applications. However, this is not implemented in this paper.

\section{B. Two prototype chips}

Our active mixer circuits use load ressitors $\mathrm{R}_{\text {load. }}$. It is frequently assumed that a poly-silicon resistor has negligible flicker noise $[1,6]$ which assumption is valid for many conventional circuits. However, in this paper the aim is at very low flicker noise mixers. The work in [11] shows that the traps at the silicon grain boundaries in the poly-silicon cause some flicker noise, resulting in a flicker noise current given by $S_{i}=\frac{\beta I^{2}}{W L} \cdot \frac{1}{f}$, where $\beta$ is a constant including technology-dependent data and temperature, $I$ is the DC current through the resistor, and $W$ and $L$ are the resistor width and length respectively. Simulation results in Fig. 11 show that without mismatch and with metal resistors, the mixer noise output only contains thermal noise (denoted by "Nominal mixer noise with MetalRload"). Including mismatch and with metal resistors, the mixer flicker noise frequency corner is below $1 \mathrm{kHz}$. Using poly resistors, even in the nominal case without any mismatch, the flicker noise of the poly-silicon resistor is dominant compared to the mixer thermal noise unless large silicon area (larger than $2100 \mathrm{um}^{2}$ ) is used. Therefore, in our design a serpentine metal resistor consisting four stacked metal layers (M1-M4) with a total area of $370 \mathrm{um}^{2}$ is used to be able to prove our concept properly.

Using the topology shown in Fig. 9 we designed two chips. One (MixerD) is optimized for full 
IM3 cancellation and partial flicker noise cancellation using the process's nominal supply voltage $\left(\mathrm{V}_{\mathrm{DD}, \mathrm{NOM}}=1.8 \mathrm{~V}\right)$. To show the robustness of the proposed flicker noise cancellation technique under the constraint of low supply voltage, a second chip (MixerNF) is optimized for full flicker noise cancellation and partial IM3 cancellation using $0.67 \times \mathrm{V}_{\mathrm{DD}, \mathrm{NOM}}(1.2 \mathrm{~V})$. The main design parameters of both circuits are listed in Table I. Since flicker noise is mainly a problem for narrowband system, the two mixer chips are designed for $0.9 \mathrm{GHz}$. Two off-chip baluns are used to generate the differential RF input and differential clock, respectively. The external differential clock signal and an on-chip LO buffer provides the LO for mixer.

\section{Comparison with other techniques}

Due to the similar topology appearance, our mixer in Fig. 9 is compared with previous techniques of flicker noise reduction. In [1] the cross-coupled pair $\mathrm{M}_{4 \mathrm{a}} / \mathrm{M}_{4 \mathrm{~b}}$ shown in Fig. 12a provides a dynamic current into the transconductor stage at the LO zero-crossings (at $\frac{T_{L O}}{2}$ ). As a result, at $\frac{T_{L O}}{2}$ the current through the switching pair and the transconductance of the switching pair is reduced. This enables a smaller $\left.v_{f l, o u t}^{M_{1} a}\right|_{\frac{T_{L O}}{2}}$ in (5) and consequently yields flicker noise reduction. The cross-coupled pair $\mathrm{M}_{4 \mathrm{a}} / \mathrm{M}_{4 \mathrm{~b}}$ turns on only around $\frac{T_{L O}}{2}$ and remains off during the remainder of the LO period, which requires a high LO voltage swing and low $\mathrm{R}_{\text {load }}(50 \mathrm{ohm}$ in [1]). To tune out the parasitic capacitance of the cross-coupled pair $\mathrm{M}_{4 \mathrm{a}} / \mathrm{M}_{4 \mathrm{~b}}$, an inductor is added to the cross-coupled pair $\mathrm{M}_{4 \mathrm{a}} / \mathrm{M}_{4 \mathrm{~b}}$ as shown in Fig. 12b in [2]. Although these dynamic bleeding techniques [1-2] and our mixer all use the cross-coupled pair $\mathrm{M}_{4 \mathrm{a}} / \mathrm{M}_{4 \mathrm{~b}}$, there are a number of fundamental differences:

- The cross-coupled pair $\mathrm{M}_{4 \mathrm{a}} / \mathrm{M}_{4 \mathrm{~b}}$ in the dynamic bleeding technique only operates around $\frac{T_{L O}}{2}$, while in our mixer the negative impedance is operational during the total LO period. As a result, our mixer only needs normal LO voltage swing, while high LO voltage swing is required in the 
dynamic bleeding technique, which may impose linearity degradation due to the switching pair (see [7]).

- The cross-coupled pair $\mathrm{M}_{4 \mathrm{a}} / \mathrm{M}_{4 \mathrm{~b}}$ in the dynamic bleeding technique is designed as a DC current injector rather than a negative resistor. Flicker noise leakage due to the finite transconductor output resistance is not addressed. The source degenerated capacitance together with the cross-coupled pair $\mathrm{M}_{4 a} / \mathrm{M}_{4 \mathrm{~b}}$ in this paper are designed as a negative impedance, which fully addresses the flicker noise leakage.

Based on the analysis in section II, in fact the mixer in Fig. $12 \mathrm{~b}$ can be made to act in the same way as our mixer if the cross-coupled pair $\mathrm{M}_{4 \mathrm{a}} / \mathrm{M}_{4 \mathrm{~b}}$ is designed to operate during the whole LO period: the cross-coupled pair together with the inductor in [2] is equivalent to the negative impedance proposed in our work. However, full flicker noise cancelling was not done in [2].

\section{SIMULATION AND MEASUREMENT}

The microphotograhps of two demonstrator mixer chips (MixerD and MixerNF) are shown in Fig. 13. The active area of the LO buffer and mixer with decap is $0.0156 \mathrm{~mm}^{2}$ for MixerD, of which $8.2 \%$ is occupied by the $Y_{\text {neg }}$ circuit. In MixerNF the $Y_{\text {neg }}$ circuit consumes $7.1 \%$ of the total active area $\left(0.014 \mathrm{~mm}^{2}\right)$. The packaged chips were measured on PCB boards for $0.9 \mathrm{GHz} \mathrm{LO}$ and $0.92 \mathrm{GHz} \mathrm{RF}$. The noise is measured by an Agilent E5500 noise measurement set-up. For noise at IF $<1 \mathrm{MHz}$ a SRS preamplifer is used to connect the mixer output with the noise set-up; for noise at IF $>1 \mathrm{MHz}$, a LeCroy AP033 active probe was used connecting the mixer output with the noise measurement set-up.

\section{A. Mixer with full-IM3/partial-flicker-noise cancellation}

For the mixer optimized for full IM3 cancellation and partial flicker noise cancellation (MixerD), the bias current of $Y_{\text {neg }}\left(I_{\text {Yneg }}\right.$ shown in Fig. 9$)$ is swept within $\pm 45 \%$ variation of the optimal value to 
demonstrate the robustness against process spread. The measured and simulated results are shown in Fig.14 as a function of the bias current normalized to the optimal value $\left(N I_{\text {Yneg }}\right)$. At the optimal bias value $\left(N I_{\text {yneg }}=1\right)$, a measured improvement of $10 \mathrm{~dB}$ for IIP3, $5 \mathrm{~dB}$ for conversion gain, $9 \mathrm{~dB}$ for DSB $\mathrm{NF} @ 1 \mathrm{kHz}$, and $1 \mathrm{~dB}$ for input $\mathrm{P}_{1 \mathrm{~dB}}$ are achieved compared to the same mixer without $\mathrm{Y}_{\text {neg. }}$. The DSB NF@10MHz degrades by $0.1 \mathrm{~dB}$. The mixer DC current increases from $9.2 \mathrm{~mA}$ to $10.7 \mathrm{~mA}$ due to the biasing of the $Y_{\text {neg }}$ circuitry, while the LO buffer current (16mA) stays unchanged. Within $\pm 45 \%$ variation of $I_{\text {Yneg, }},>5 \mathrm{~dB}$ gain improvement, $>6 \mathrm{~dB} \mathrm{NF} @ 1 \mathrm{kHz}$ reduction, $<0.2 \mathrm{~dB}$ thermal NF degradation, no input $\mathrm{P}_{1 \mathrm{~dB}}$ degradation are achieved. Fig. 14b shows the flicker NF reduction at a very low frequency $(1 \mathrm{kHz})$, where the flicker noise is dominant and the thermal noise can be neglected. Fig. 15 shows the measured fundamental and IM3 output at the optimal bias value $\left(N I_{\text {Yneg }}=1\right)$. Due to higher-order nonlinearity distortion introduced by $\mathrm{Y}_{\text {neg }}$, the IM3 curve start to show $5^{\text {th }}$ order behavior for Pin $>-18 \mathrm{dBm}$. The measured mixer DSB NF is shown in Fig. 16. The spikes are from the equipment power supply and the measurement setup. Although $\mathrm{Y}_{\text {neg }}$ introduces $5 \mathrm{~dB}$ thermal noise to the mixer, $5 \mathrm{~dB}$ more gain also provided by $\mathrm{Y}_{\text {neg }}$ lowers the input-referred noise of the $\mathrm{R}_{\text {load }}$ by $5 \mathrm{~dB}$ and results in overall less than $0.1 \mathrm{~dB}$ degradation in the thermal noise figure. The flicker noise corner frequency decreases from $100 \mathrm{kHz}$ to $20 \mathrm{kHz}$.

Simulated and measured effects of mismatch and process spread on NF and IIP3 of MixerD are shown in Fig. 17. The results of a 200-time Monte Carlo simulation using a realistic production variation model for device mismatches and process spread, in Fig. 17a, show a mean DSB NF@1kHz of $19.2 \mathrm{~dB}$ (nominally $19 \mathrm{~dB}$ ) which is about $7 \mathrm{~dB}$ lower than the mixer without $\mathrm{Y}_{\text {neg, }}$, while the measurements of ten dies from one wafer show $+7 \mathrm{~dB}$ NF reduction at $1 \mathrm{kHz}$ in Fig. 17b. Note that such a high NF is not the result a badly designed mixer but is due to the very low frequency $(1 \mathrm{kHz})$, 
where the flicker noise is significant. In comparison, the measured NF@1kHz of the low-flicker-noise mixer in [2] is $29 \mathrm{~dB}$. In Fig. $17 \mathrm{c}$ a 200 -time Monte Carlo simulation shows a mean IIP3 of $10.8 \mathrm{dBm}$ (nominally $12 \mathrm{dBm}$ ) which is $9 \mathrm{~dB}$ higher than the mixer without $\mathrm{Y}_{\text {neg, }}$, whereas more than $6 \mathrm{~dB}$ IIP3 improvement is measured in ten dies from one wafer as shown in Fig. 17d. For the temperature range $\left[-40^{\circ} \mathrm{C}\right.$ to $\left.80^{\circ} \mathrm{C}\right]$ in the nominal corner, simulations show $>6.7 \mathrm{~dB}$ flicker NF reduction in Fig. $18 \mathrm{a}$; the IM3 cancellation becomes less effective as the temperature increases as shown in Fig. 18b. We did not implement a control loop to adjust the negative impedance over temperature; the realized chips aim to prove the principle of flicker noise and distortion cancellation.

\section{B. Mixer with full-flicker-noise / partial-IM3 cancellation}

For the mixer optimized for full flicker noise cancellation (MixerNF), Fig.19 shows the measured and simulated results as a function of the bias current for $\mathrm{Y}_{\text {neg }}$ normalized to the optimal value $\left(N I_{\text {Yneg }}\right)$. When $\mathrm{Y}_{\text {neg }}$ is not enabled, MixerNF has about 5dB less gain compared with MixerD. There is less voltage swing across the transistors' terminals resulting less distortion and hence higher IIP3. At the optimal bias value $\left(N I_{\text {yneg }}=1\right)$ a measured improvement of $8 \mathrm{~dB}$ for DSB NF@1kHz, $1.4 \mathrm{~dB}$ for conversion gain, $0.1 \mathrm{~dB}$ for the DSB NF@5MHz and $2.5 \mathrm{~dB}$ for input $\mathrm{P}_{1 \mathrm{~dB}}$ are achieved compared to the same mixer without $Y_{\text {neg. }}$. The mixer DC current increases by $4 \%$ due to the biasing of the $Y_{\text {neg }}$ circuitry, while the LO buffer current (4.8mA) stays unchanged. The difference between the measured and simulated IIP3 shown in Fig. 19d may be due to the fact that this mixer is operated at low supply voltage $\left(0.67 \times V_{D D, N O M}\right)$, where the headroom for the $Y_{\text {neg }}$ circuit is insufficient to provide a robust IM3 cancellation. Full flicker noise cancellation at the optimal bias value $\left(N I_{\text {yneg }}=1\right)$ shown in Fig. 19(a) suggested by simulation is not found in measurement, probably due to external low-frequency noise contributed by the measurement set-up and due to the LO phase noise leakage resulting from 
mismatches in the mixer. However, Fig. 19a shows that more than $10 \mathrm{~dB}$ improvement for the flicker NF can be achieved for very broad bias range (for $N I_{Y n e g}>1.75$ ). The robustness of this flicker noise cancellation under low supply voltage is estimated for $N I_{\text {Yneg }}=2$ in MixerNF. Fig. 20a shows the results of a 200-time Monte Carlo with mismatch and process spread, indicating a mean DSB@1kHz of $21.9 \mathrm{~dB}$ (nominally $20 \mathrm{~dB}$ ) which is $15 \mathrm{~dB}$ lower than in the mixer without $\mathrm{Y}_{\text {neg; }}$; measurements on ten dies shows more than $11 \mathrm{~dB}$ flicker NF reduction, see Fig. $20 \mathrm{~b}$. For the temperature range $\left[-40^{\circ} \mathrm{C}\right.$ to $\left.80^{\circ} \mathrm{C}\right]$ in the nominal corner, simulations shows more than $14 \mathrm{~dB}$ flicker NF reduction, see Fig. 21 . The measured mixer noise output is shown in Fig. 22 (for $\mathrm{Y}_{\text {neg }}$ biased at $N I_{\text {yneg }}=2$ ). The flicker corner frequency decreases from $200 \mathrm{kHz}$ to $20 \mathrm{kHz}$; the rolling-off behavior for IF $>5 \mathrm{MHz}$ is due to the IF filter in the measurement setup.

\section{Benchmarking}

The mixer with full-IM3/partial-flicker noise cancellation (MixerD) presented in this paper is compared with previous works on flicker noise reduction [1-4] in Table II. Since the flicker NF value depends on a few factors such as circuit bias and technology-related flicker noise corner, our technique is compared with previous works in term of the value of flicker noise reduction. It shows that the presented technique provides very good flicker NF reduction, while at the same time it achieves the largest improvement in IIP3 and gain without using on-chip inductors or high supply voltages or increasing the LO power. In conclusion, this flicker noise/IM3 cancellation provides solutions for reducing flicker noise and improving linearity of CMOS active mixers.

\section{CONCLUSION}

A new technique providing simultaneous cancellation of flicker noise and IM3 distortion for active mixers is presented without using on-chip inductors or high supply voltages or increasing the 
LO power. By using a negative impedance $\left(\mathrm{Y}_{\text {neg }}\right)$, the flicker noise leakage from the switching pairs is minimized. Meanwhile the negative impedance enables IM3 distortion cancellation between the switching pairs and the transconductor stage, which yields overall IM3 improvement. The techniques also improve the conversion gain while it has little effect on the thermal noise. For the demonstrator mixer chip optimized for full-IM3/partial-flicker-noise cancellation, 9dB flicker noise suppression, $10 \mathrm{~dB}$ improvement for IIP3, $5 \mathrm{~dB}$ improvement for conversion gain and $1 \mathrm{~dB}$ improvement for input $P_{1 d B}$ are achieved. The $Y_{\text {neg }}$ circuit increases the thermal NF by $0.1 \mathrm{~dB}$, power consumption by $16 \%$ and active area by $8 \%$. Under mismatch and process spread, a 200-time Monte Carlo simulation shows $7 \mathrm{~dB}$ reduction in mean $\mathrm{NF} @ 1 \mathrm{kHz}$ and $9 \mathrm{~dB}$ increase in mean IIP3. A ten-sample measurement shows over 7dB reduction in NF@1kHz and more than 6dB increase in IIP3. Simulations indicate that the flicker noise cancellation is not very sensitive to temperature variation $\left[-40^{\circ} \mathrm{C}\right.$ to $\left.80^{\circ} \mathrm{C}\right]$, while the IM3 cancellation degrades as the temperature increases. For the demonstrator mixer chip optimized for full-flicker-noise/partial-IM3 cancellation under low supply voltage $\left(0.67 \times \mathrm{V}_{\mathrm{DD}, \mathrm{NOM}}\right)$, more than $10 \mathrm{~dB}$ flicker noise suppression is measured within $\pm 200 \%$ variation of the negative impedance bias current. The ten-sample measurement shows over $11 \mathrm{~dB}$ flicker NF reduction, and the simulation shows more than $14 \mathrm{~dB}$ flicker NF reduction for the temperature range $\left[-40^{\circ} \mathrm{C}\right.$ to $\left.80^{\circ} \mathrm{C}\right]$.

\section{Acknowledgements:}

We thank NXP Semiconductors for chip fabrication, and G. van der Weide, M. C. M. Soer and H. de Vries for their help. 


\section{APPENDIX}

The real part of (6) is given by

$$
\operatorname{Re}\left(\left.v_{f l, \text { out }}^{M_{1 a}}\right|_{t_{1}}\right)=\frac{-g_{m}^{M_{1} R_{R}}\left[G^{M_{3}}\left(g_{m}^{M_{1} a}+G^{M_{3}}\right)+\left(\omega_{L O} C^{M_{3}}\right)^{2}\right] v_{f l, \text { in }}^{M_{1} a}}{\left(g_{m}^{M_{1} a}+G^{M_{3}}\right)^{2}+\left(\omega_{L O} C^{M_{3}}\right)^{2}}
$$

where $G^{M_{3}}=g_{d s}^{M_{3 a}}+2 G_{n e g}$ and $C^{M_{3}}=C_{\text {tail }}+2 C_{n e g}$.

When $\left\{\begin{array}{l}G_{n e g}>\frac{1}{4}\left(-2 g_{d s}^{M_{3 a}}-g_{m}^{M_{1 a}}-\sqrt{g_{m}^{M_{1 a}{ }^{2}}-\left(2 \omega_{L O} C^{M_{3}}\right)^{2}}\right) \\ G_{n e g}<\frac{1}{4}\left(-2 g_{d s}^{M_{3 a}}-g_{m}^{M_{1 a}}+\sqrt{g_{m}^{M_{1 a}{ }^{2}}-\left(2 \omega_{L O} C^{M_{3}}\right)^{2}}\right)\end{array}\right.$

the real part of (6) is positive. For $C_{\text {neg }} \approx-0.5 C_{\text {tail }}, C^{M_{3}} \approx 0$ and (A2) can be simplified to

$$
\frac{-g_{d s}^{M_{3 a}}-g_{m}^{M_{1 a}}}{2}<G_{n e g}<\frac{-g_{d s}^{M_{3 a}}}{2}
$$

Therefore, for $G_{n e g} \in\left[\frac{-g_{d s}^{M_{3} a}-g_{m}^{M_{1} a}}{2}, \frac{-g_{d s}^{M_{3} a}}{2}\right]$ the real part of (6) is positive.

\section{REFERENCES}

[1] H. Darabi and J. Chiu, "A noise cancellation technique in active RF-CMOS mixers," IEEE J. Solid-State Circuits, vol. 40, pp. 2628-2632, Dec. 2005.

[2] J. Yoon, H. Kim, C. Park, J. Yang, H. Song, S. Lee and B. Kim, “A new RF CMOS Gilbert mixer with improved noise figure and linearity,” IEEE Trans. Microwave Theory and Techniques, vol. 56, pp. 626-631, Mar. 2008.

[3] R. S. Pullela, T. Sowlati and D. Rozenblit, "Low flicker-Noise quadrature mixer topology," IEEE Int. Solid-State Circuits Conf. (ISSCC) Dig. Tech. Papers, San Francisco, CA, 2006, pp. 1870 - 1879.

[4] J. Park, C. H. Lee, B.-S Kim and J. Laskar, "Design and analysis of low flicker-noise CMOS mixers for direct-conversion receivers", IEEE Trans. Microwave Theory and Techniques, vol. 54, pp. 4372-4380, Dec. 2006.

[5] B. Razavi, "Design considerations for future RF circuits", Proc. IEEE ISCAS, May 2008.

[6] H. Darabi and A. A. Abidi, "Noise in RF-CMOS mixers: a simple physical model," IEEE J. Solid-State Circuits, vol. 35, pp. 15-25, Jan. 2000.

[7] W. Cheng, A. J. Annema, J. A. Croon and B. Nauta, "Noise and nonlinearity modeling of active mixers for fast and accurate estimation", IEEE Trans. Circuits and Systems I, vol. 58, pp. 276-289, Feb. 2011.

[8] C. C. Enz and G. C. Temes, "Circuit techniques for reducing the effects of op-amp imperfections: Autozeroing, correlated double sampling, and chopper stabilization," Proceedings of the IEEE, vol. 84, no. 11, pp. 1584-1615, Nov. 1996.

[9] C. Tilhac, S. Razafimandimby, A. Cathelin, S. Bila, V. Madrangeas and D. Belot, "A Tunable bandpass BAE-filter architecture using negative capacitance circuitry," IEEE Radio Frequency integrated Circuits (RFIC)Symposium, pp.605 - 608, 2008.

[10] J. C. Zhan, K. Maurice, J. Duster and K. T. Kornegay, "Analysis and design of negative impedance LC oscillators using bipolar transistors," IEEE Trans. Circuits and Systems I, vol. 50, pp. 1461-1464, Nov. 2003.

[11] R. Brederlow, W. Weber, C. Dahl, D. Schmitt-Landsiedel and R. Thewes, "Low-frequency noise of integrated poly-silicon resistors," IEEE Trans. Electron Devices, vol. 48,nl.6, pp. 1180-1187, Nov. 2001. 


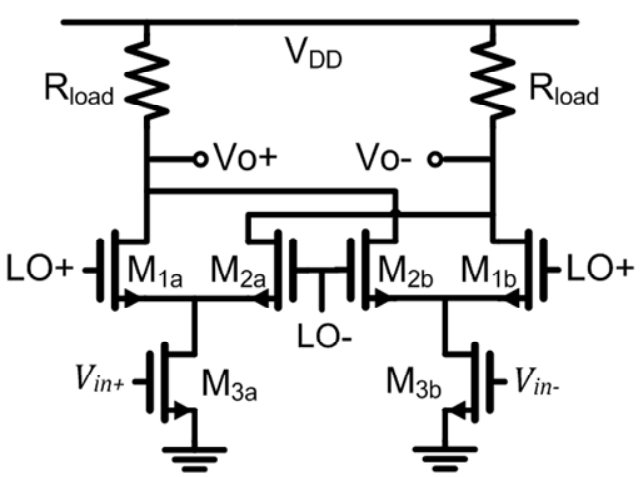

(a)

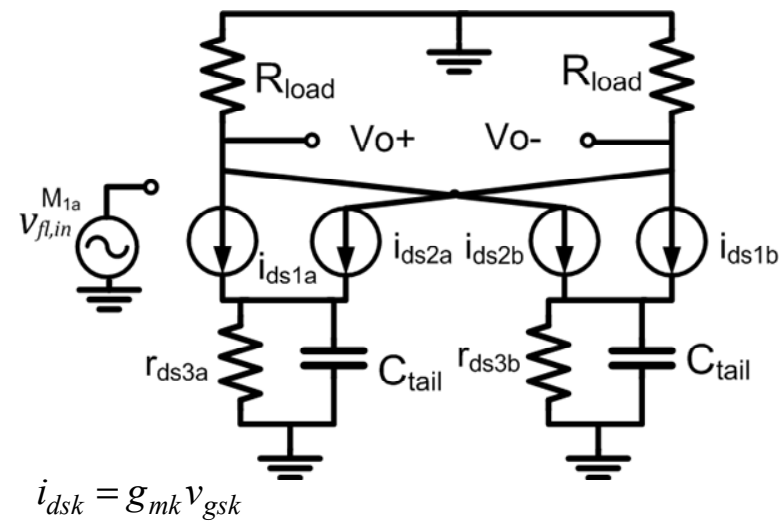

(b)

Fig. 1. (a) Schematic and (b) time-varying noise model of the double-balanced Gilbert mixer.

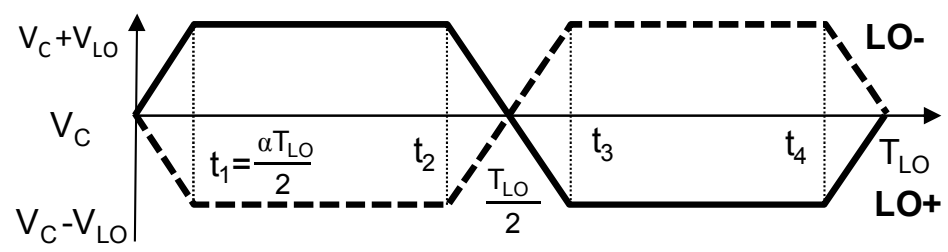

(a)

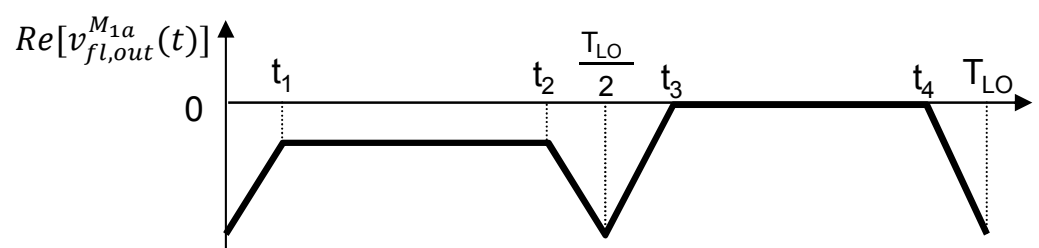

(b)

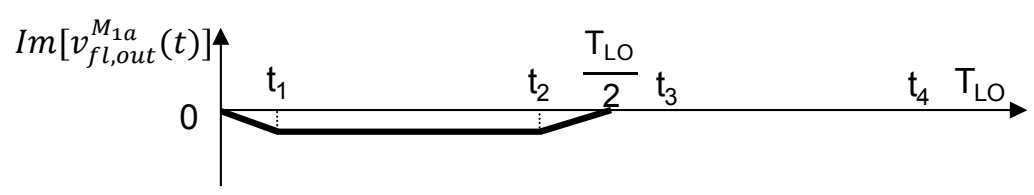

(c)

Fig. 2. (a) Waveform of the LO signal, (b) and (c): approximation of the real and imaginary part of $v_{f l, o u t}^{M_{1 a}}(t)$ respectively. 


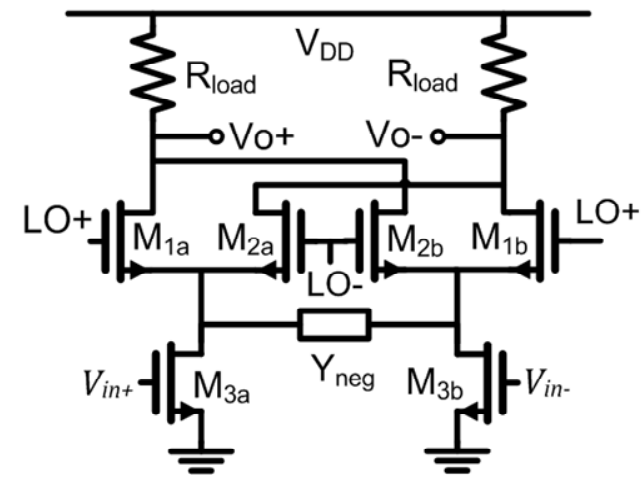

(a)

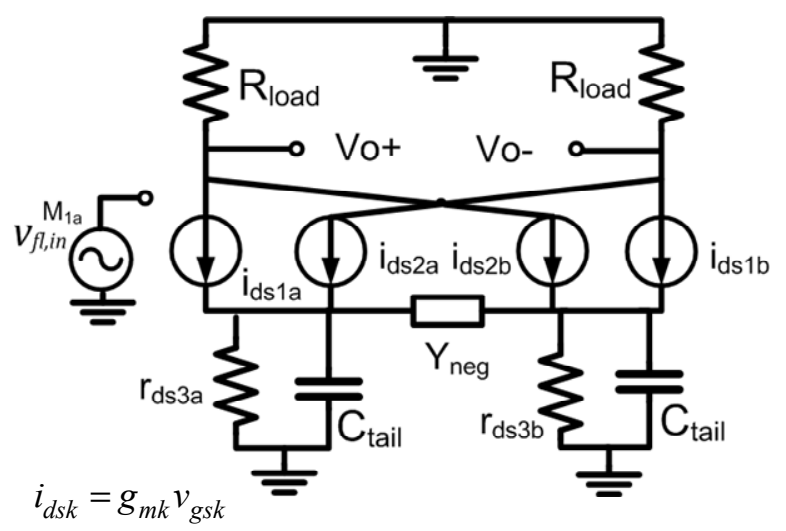

(b)

Fig. 3. (a) Schematic and (b) time-varying noise model for the mixer with a negative impedance for flicker noise cancellation.

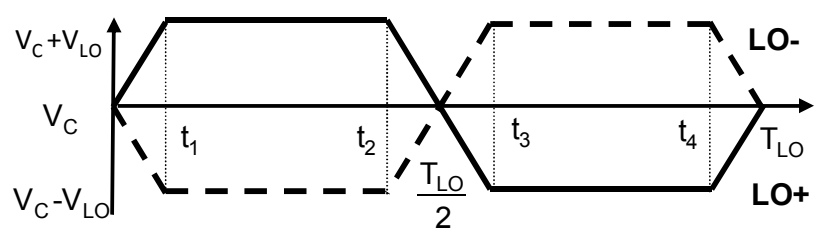

(a)

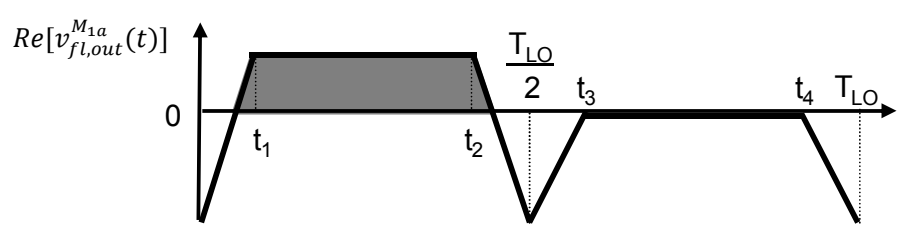

(b)

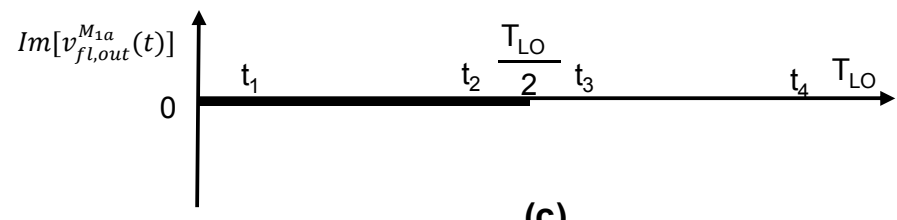

(c)

Fig. 4. (a) Waveform of the LO signal. (b) and (c): approximation of the real respectively imaginary part of $v_{f l, o u t}^{M_{1} a}(t)$ using a negative impedance for flicker noise cancellation. 


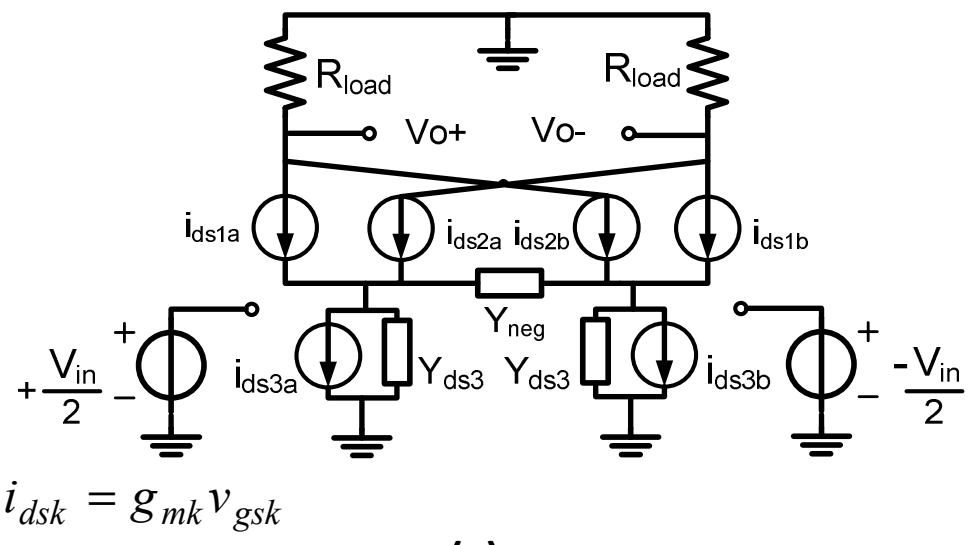

(a)

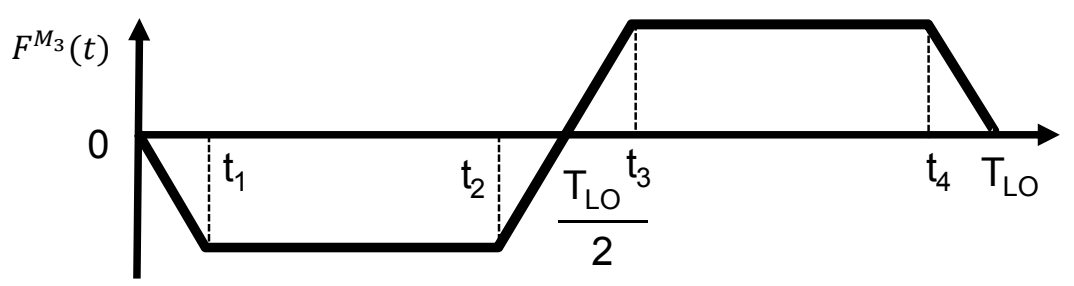

(b)

Fig. 5. (a) Time-varying linear model for calculating the voltage gain of the mixer with a negative impedance for flicker noise cancellation and (b) the approximation for the instantaneous voltage gain $F^{M_{3}}(t)$.

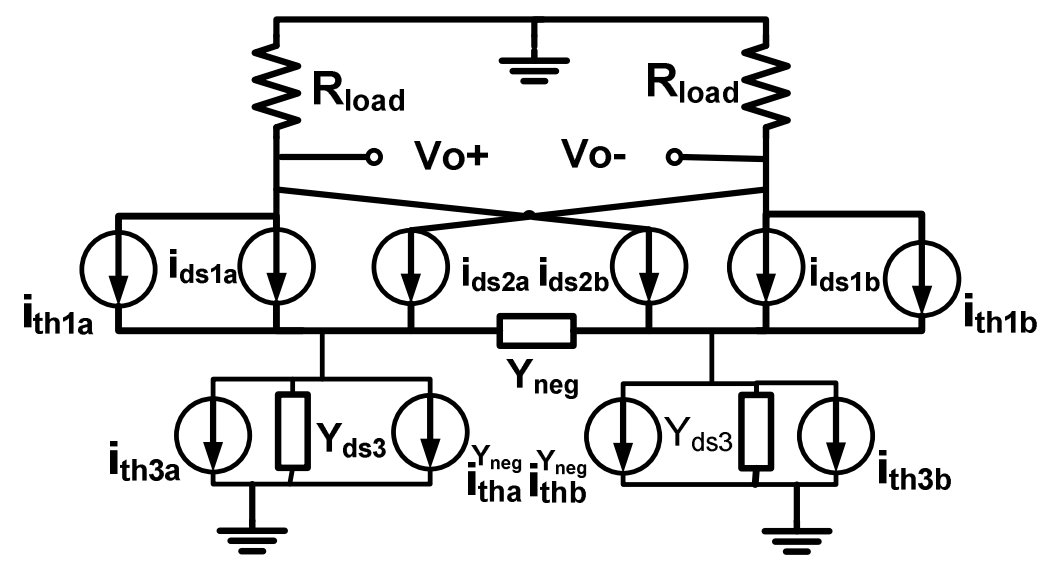

Fig. 6. Time-varying noise model for calculating the thermal noise of the mixer with a negative impedance. 


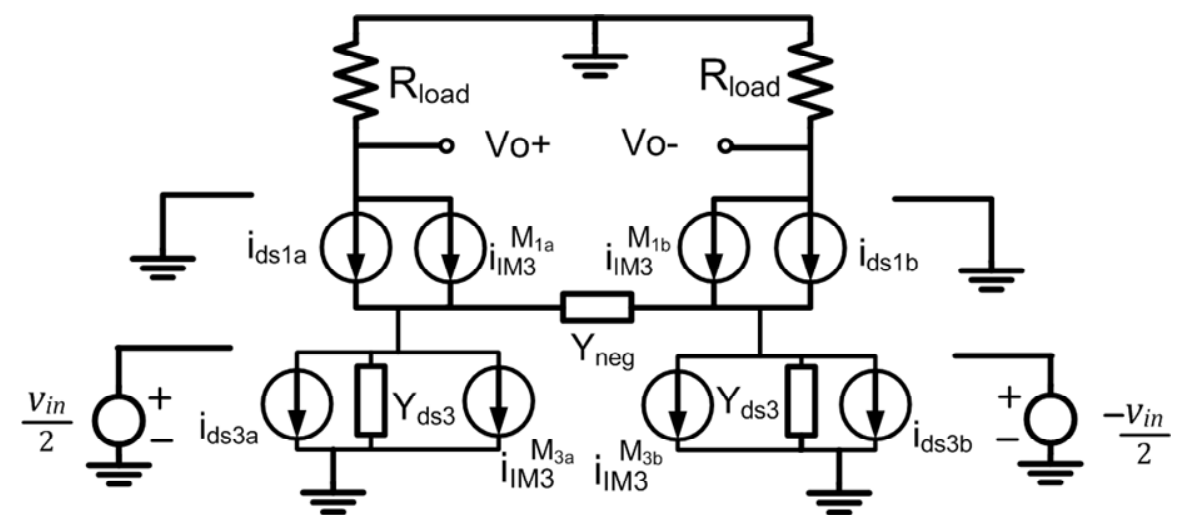

Fig. 7. Circuit model for the mixer distortion analysis.

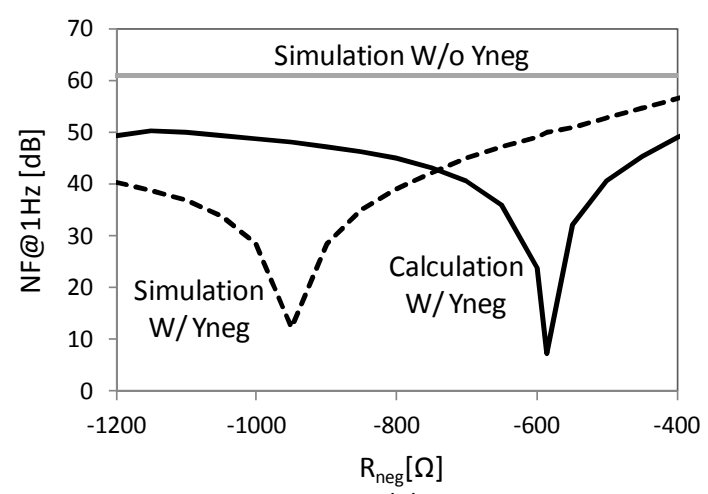

(a)

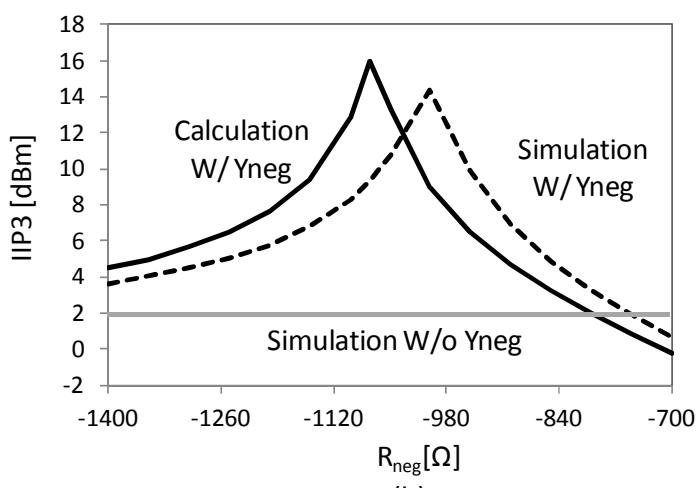

(b)

Fig. 8. Simulated and calculated (a) NF at $1 \mathrm{~Hz}$ and (b) IIP3 as a function of the negative resistance for the mixer shown in Fig. 3(a) at an LO of $0.9 \mathrm{GHz}$.

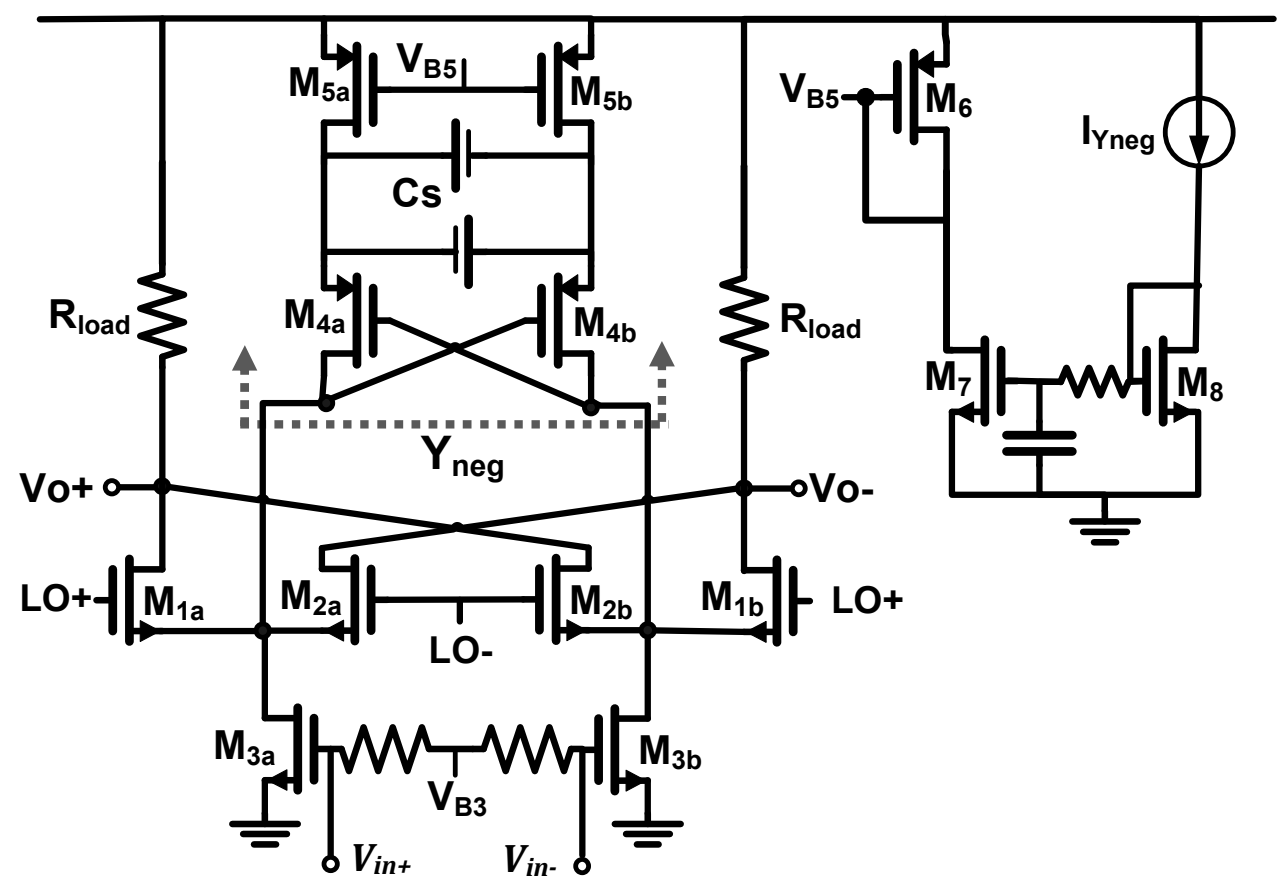

Fig. 9. The schematic of the mixer with a negative impedance $Y_{\text {neg. }}$. 


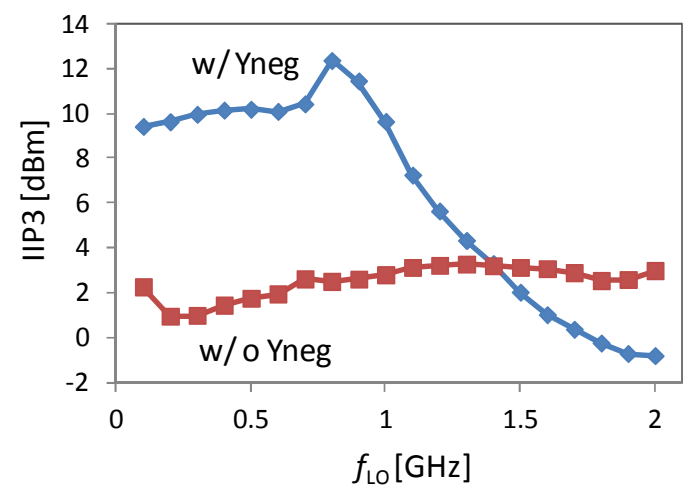

(a)

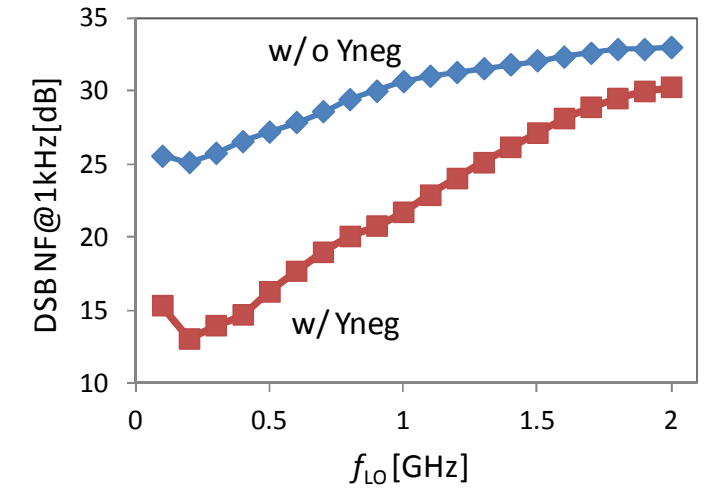

(b)

Fig. 10. The simulated (a) IIP3 and (b) DSB NF at $1 \mathrm{kHz}$ as a function of LO frequency for MixerD with and without using the negative impedance. MixerD is designed for full IM3 cancellation and partial flicker noise cancellation at $0.9 \mathrm{GHz} \mathrm{LO}$.

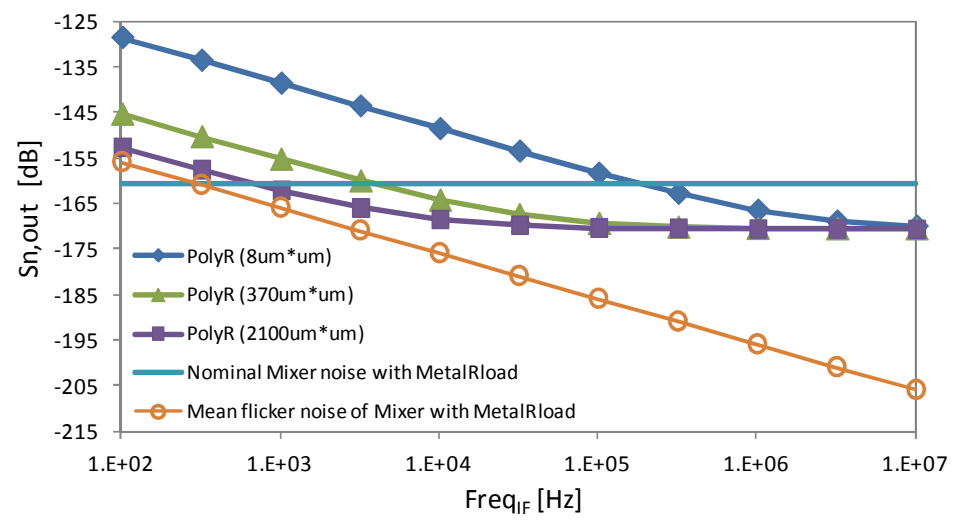

Fig. 11. The simulated output noise of the mixer using poly-silicon resistor and metal resistor as $\mathrm{R}_{\text {load. }}$.

Table I: Main design parameters for two prototype chips (MixerD and MixerNF)

\begin{tabular}{|c|c|c|c|c|c|c|c|}
\hline Chip name & $\begin{array}{c}\mathrm{W} / \mathrm{L}[\mu \mathrm{m}] \\
\left(\mathrm{M}_{1} / \mathrm{M}_{2}\right)\end{array}$ & $\begin{array}{c}\mathrm{W} / \mathrm{L}[\mu \mathrm{m}] \\
\left(\mathrm{M}_{3}\right)\end{array}$ & $\begin{array}{c}\mathrm{W} / \mathrm{L}[\mu \mathrm{m}] \\
\left(\mathrm{M}_{4}\right)\end{array}$ & $\begin{array}{c}\mathrm{W} / \mathrm{L}[\mu \mathrm{m}] \\
\left(\mathrm{M}_{5}\right)\end{array}$ & $\begin{array}{c}\mathrm{R}_{\text {load }} \\
{[\Omega]}\end{array}$ & $\begin{array}{c}\mathrm{C}_{\mathrm{s}} \\
{[\mathrm{pF}]}\end{array}$ & $\begin{array}{c}\mathrm{V}_{\mathrm{dd}} \\
{[\mathrm{V}]}\end{array}$ \\
\hline MixerD & $100 / 0.16$ & $80 / 0.16$ & $80 / 0.16$ & $104 / 0.16$ & 250 & 1.2 & 1.8 \\
\hline MixerNF & $108 / 0.16$ & $72 / 0.16$ & $68 / 0.18$ & $68 / 0.18$ & 250 & 0.8 & 1.2 \\
\hline
\end{tabular}

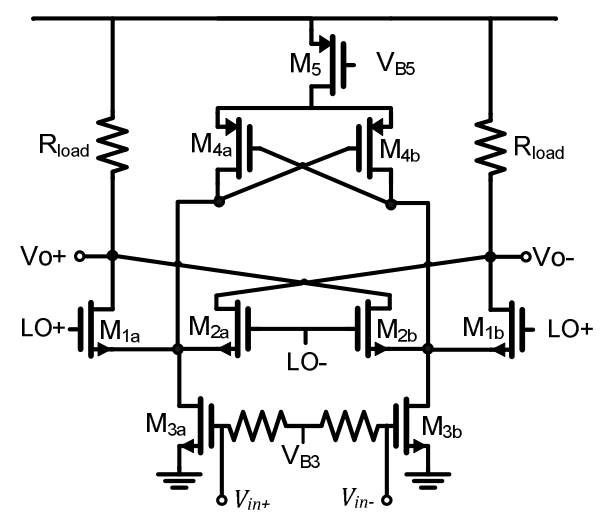

(a)

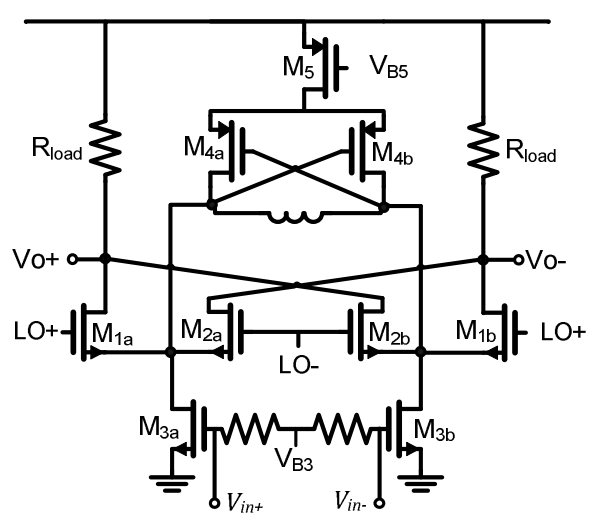

(b) 
Fig. 12 Two mixers for flicker noise reduction. a) Dynamic bleeding [1], b) Dynamic bleeding with a inductor [2].

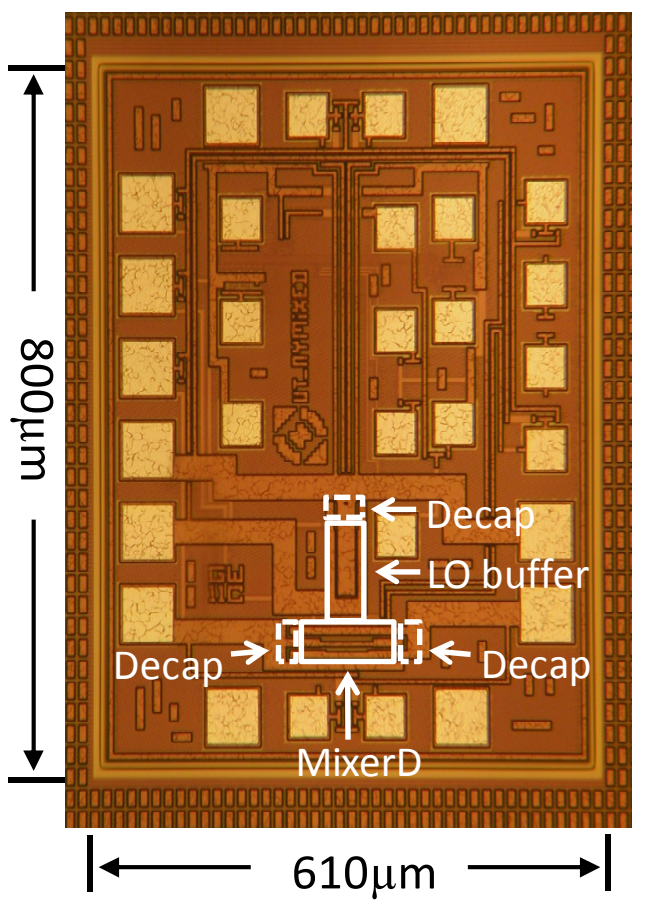

(a)

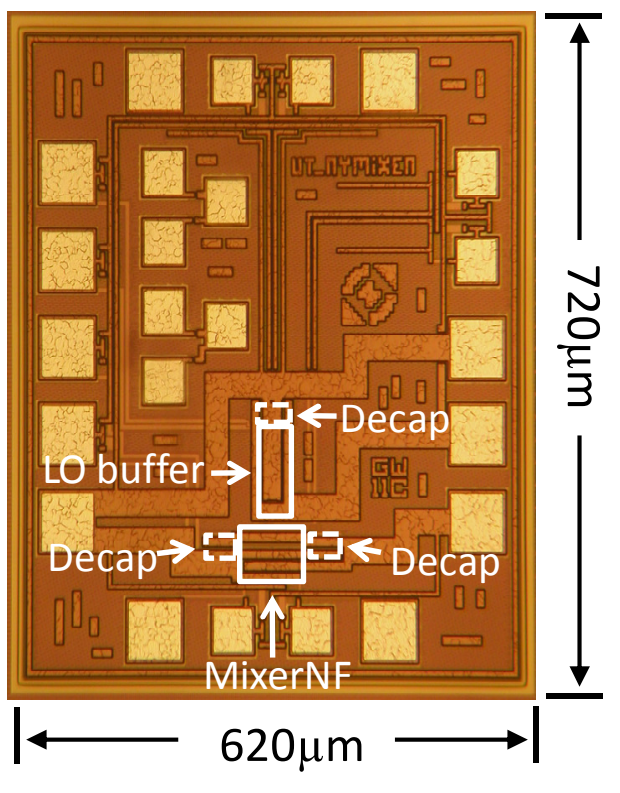

(b)

Fig. 13. Chip photo of (a) MixerD and (b) MixerNF.

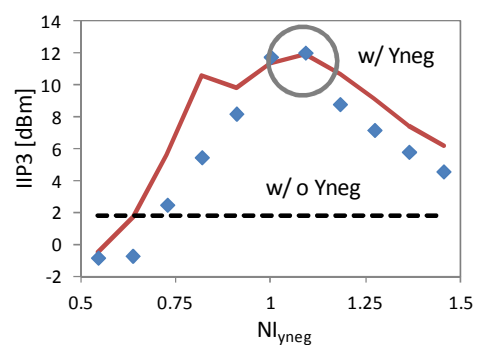

(a)

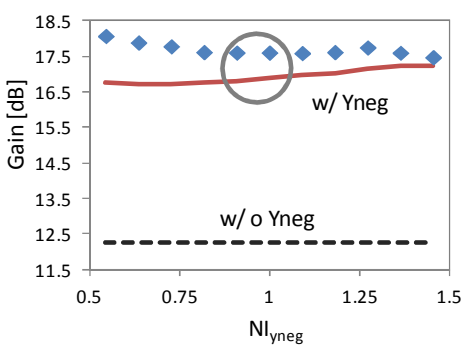

(d)

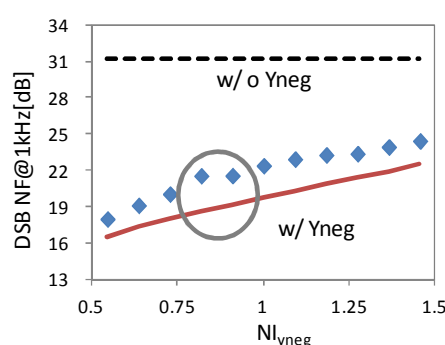

(b)

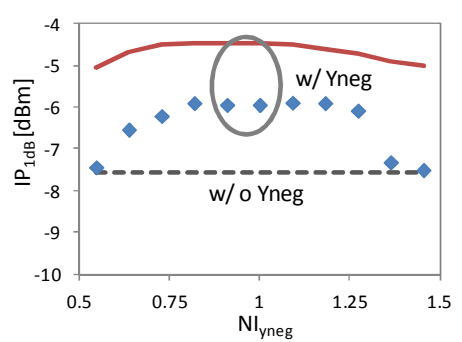

(e)

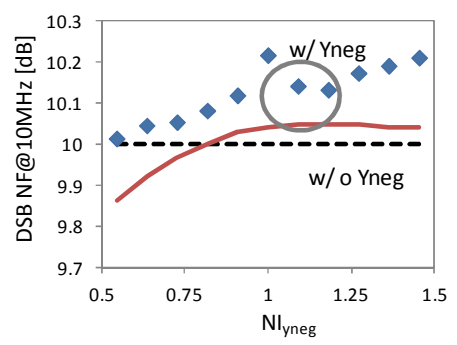

(c)

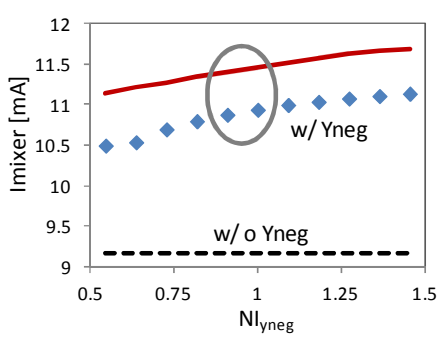

(f)

Fig. 14. IIP3, NF, conversion gain, input $\mathrm{P}_{1 \mathrm{~dB}}$ and dc current taken by the mixer as a function of the normalized bias current of $\mathrm{Y}_{\text {neg }}$ for MixerD. Solid line for simulated results and symbol for measured results of the mixer with $Y_{\text {neg. }}$. Dashed line for measured results of the mixer without $Y_{\text {neg. }}$. 


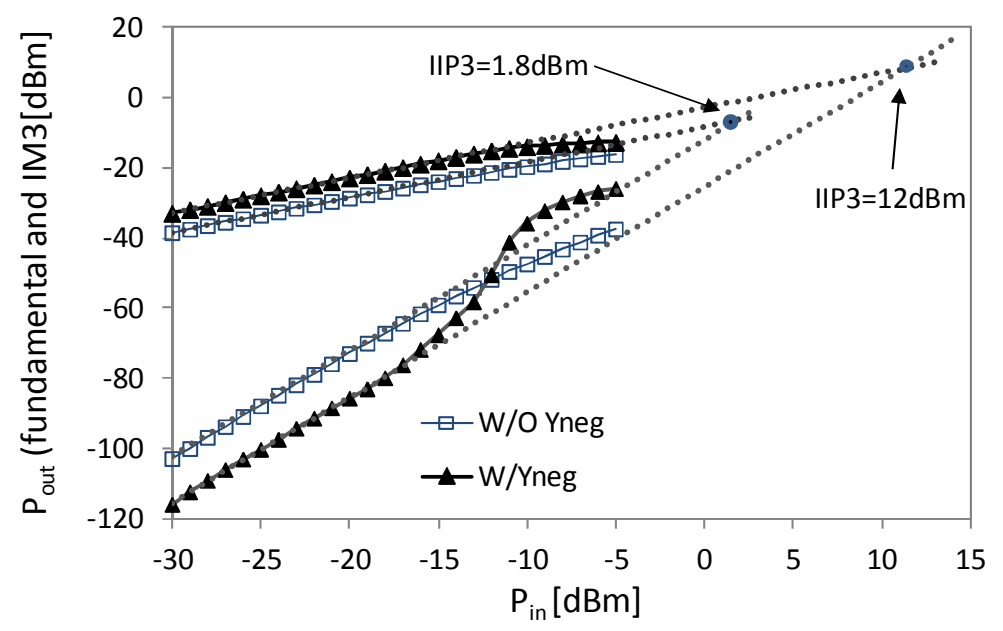

Fig. 15. Measured fundamental and IM3 output vs input power $P_{\text {in }}$ for MixerD.

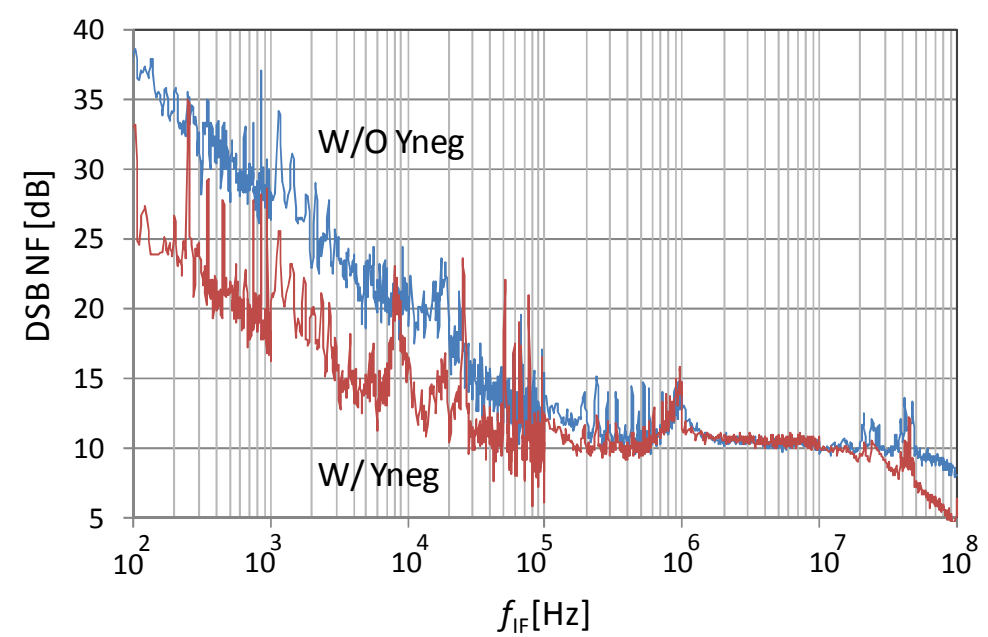

Fig. 16. Measured DSB NF for mixer (MixerD) with and without using $Y_{\text {neg. }}$.

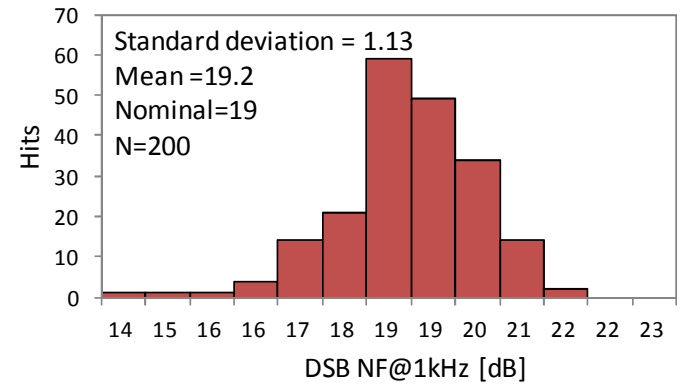

(a)

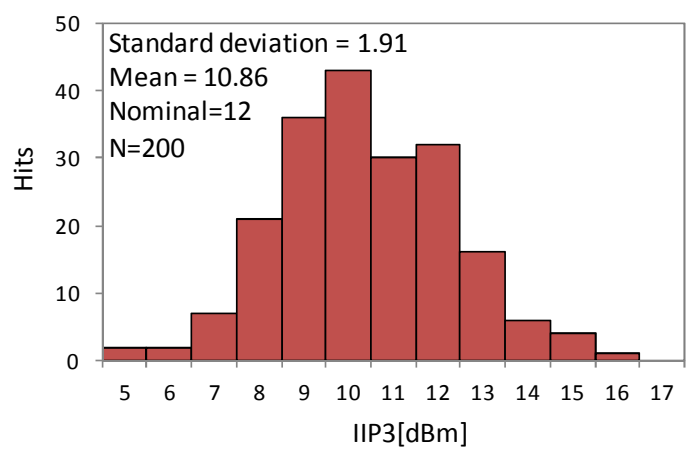

(c)

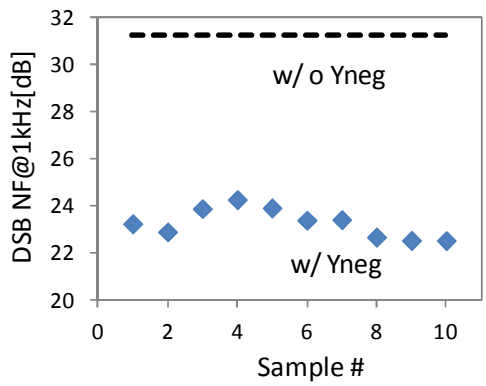

(b)

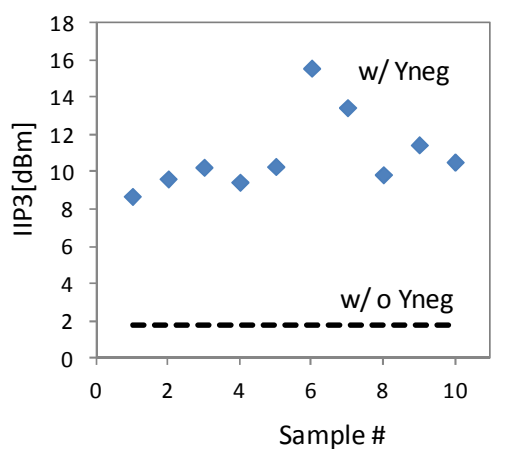

(d) 
Fig. 17. Effect of mismatches and process spread on NF and IIP3 of MixerD with $Y_{\text {neg }}\left(N I_{\text {Yneg }}=1\right)$. (a) 200-time Monte Carlo simulation results of DSB NF@1kHz (b) measured DSB NF@1kHz of ten dies, (c) 200-time Monte Carlo simulation results of IIP3, (d) measured IIP3 of ten dies. Symbol for measured results of the mixer with $Y_{\text {neg. }}$. Dashed line for measured results of one mixer sample without $Y_{\text {neg. }}$

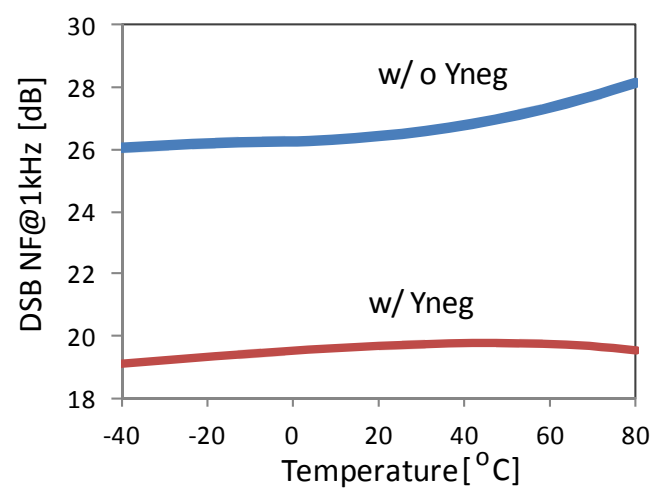

(a)

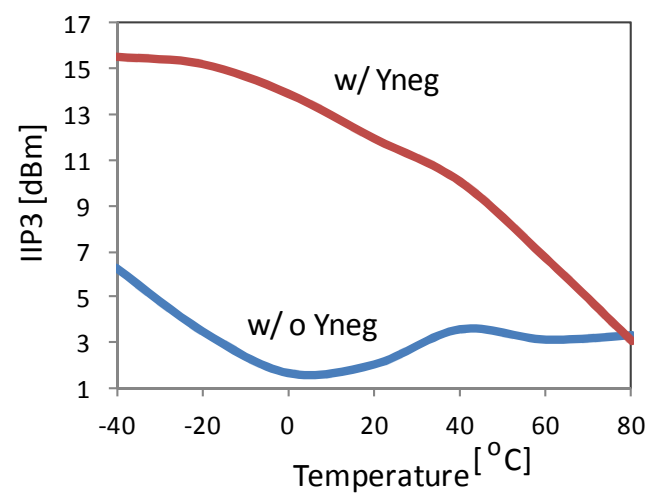

(b)

Fig. 18. Simulated NF and IIP3 for mixer (MixerD) with and without using $\mathrm{Y}_{\text {neg }}$ as a function of temperature.

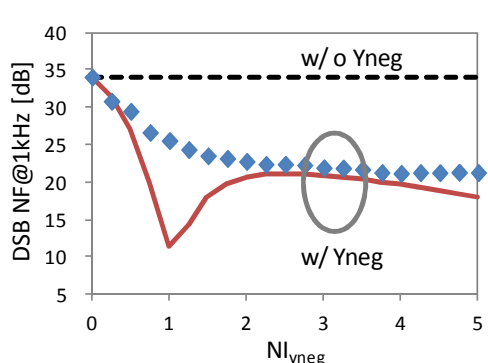

(a)

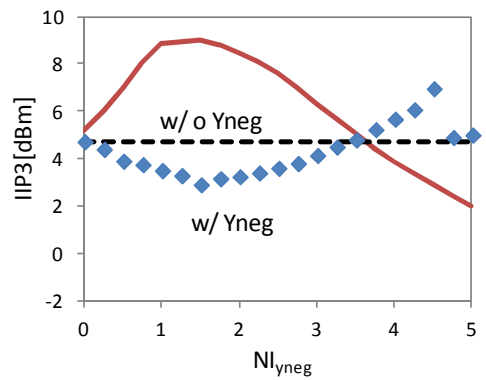

(d)

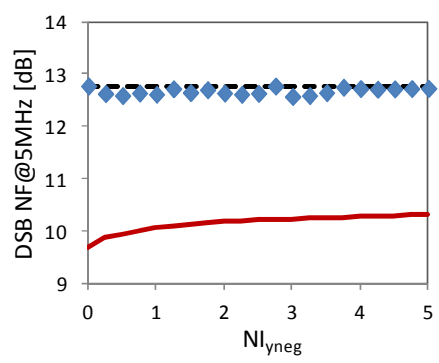

(b)

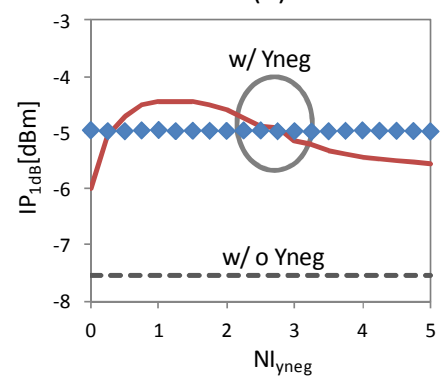

(e)

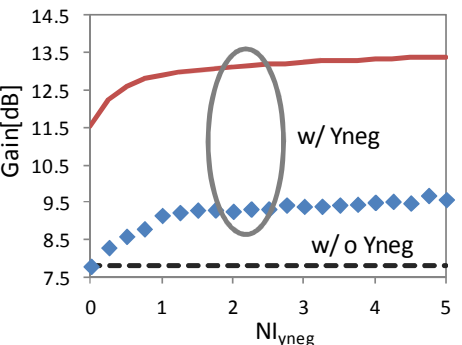

(c)

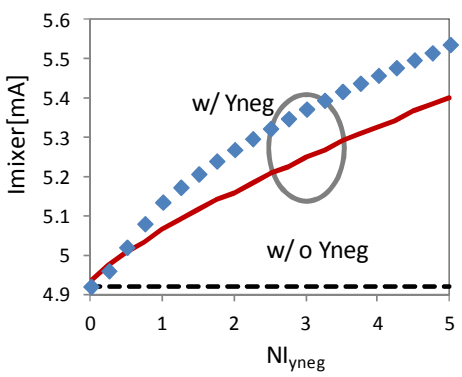

(f)

Fig. 19. NF, IIP3, conversion gain, input $\mathrm{P}_{1 \mathrm{~dB}}$ and dc current taken by the mixer as a function of the normalized bias current of $\mathrm{Y}_{\text {neg }}$ for MixerNF. Solid line for simulated results and symbol for measured results of the mixer with $Y_{\text {neg. }}$. Dashed line for measured results of the mixer without $Y_{\text {neg. }}$. 


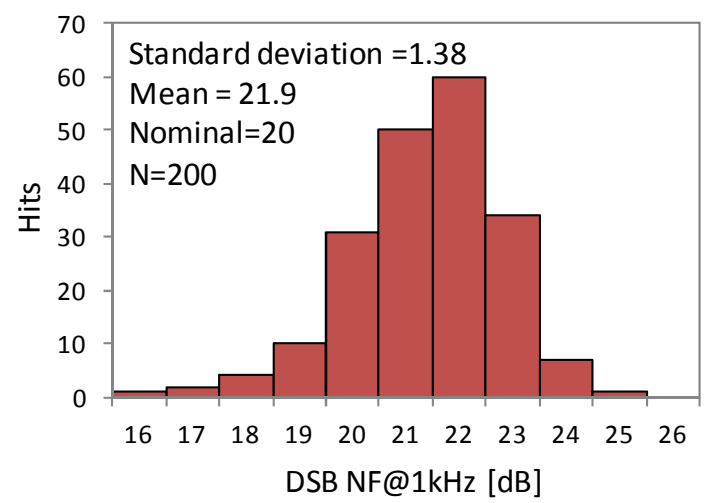

(a)

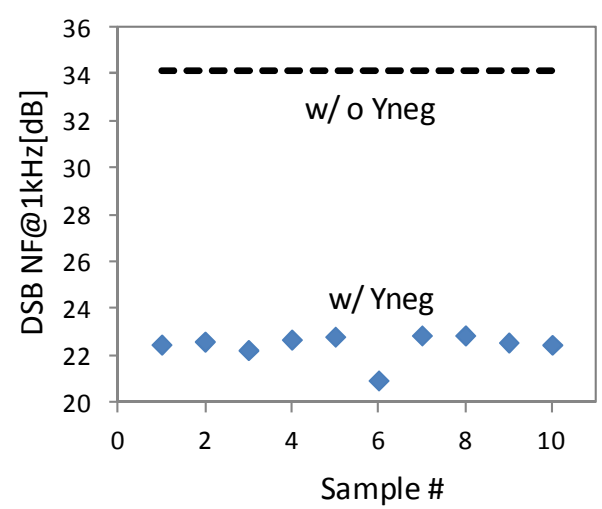

(b)

Fig. 20. (a) 200-time Monte Carlo simulation results of DSB NF@1kHz for MixerNF (NI $\left.I_{\text {yneg }}=2\right)$. (b) Measured DSB NF@1 $1 \mathrm{kHz}$ for MixerNF $\left(N I_{\text {yneg }}=2\right)$ of ten dies.

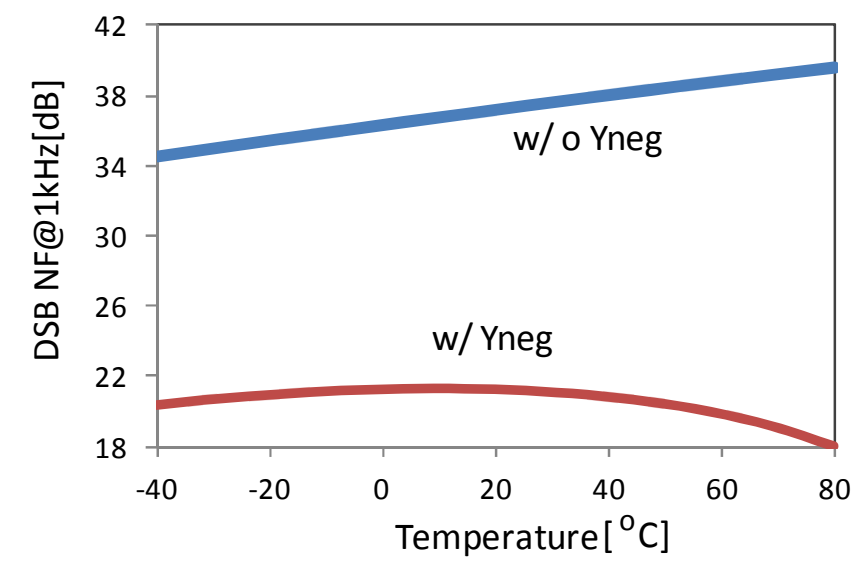

Fig. 21. Simulated NF for mixer (MixerNF) with and without using $Y_{\text {neg }}$ as a function of temperature.

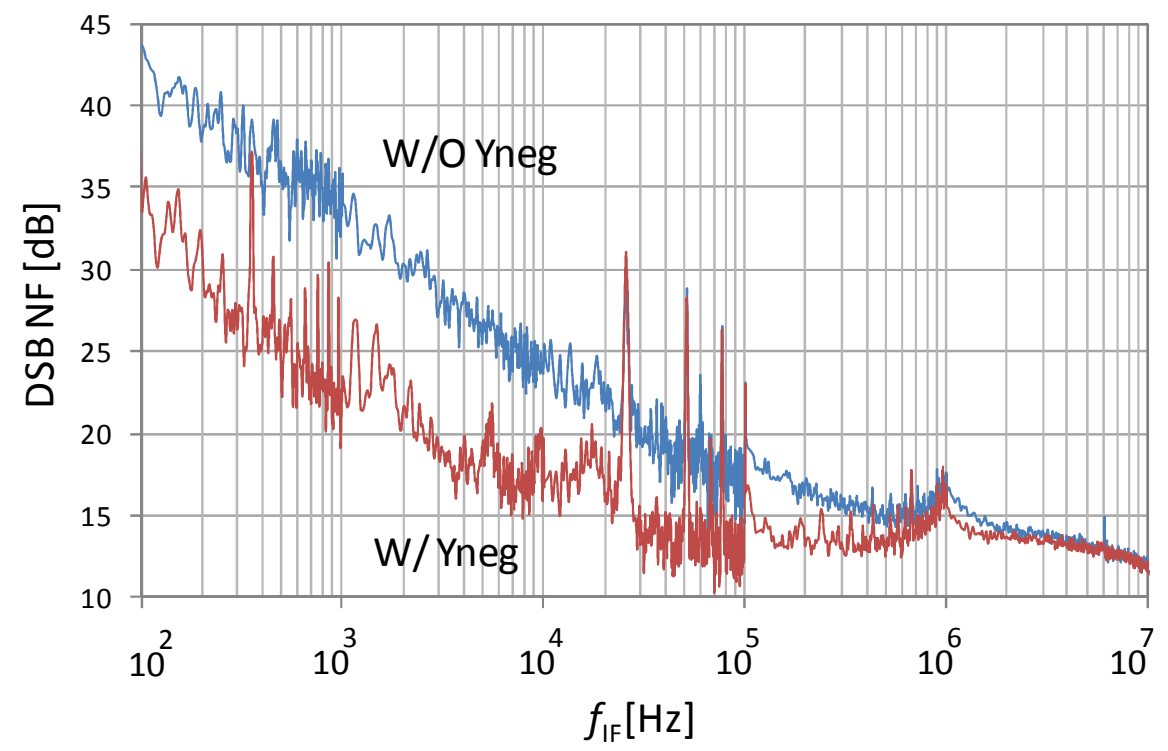

Fig. 22. Measured DSB NF for mixer with and with using $Y_{\text {neg }}$ for MixerNF $\left(N I_{y n e g}=2\right)$. 
Table II. Comparison of techniques for flicker noise reduction in CMOS active mixers.

\begin{tabular}{|c|c|c|c|c|c|c|}
\hline & & Darabi[1] & Yoon[2] & Park[4] & Pullela[3] & $\begin{array}{l}\text { This } \\
\text { work }\end{array}$ \\
\hline \multicolumn{2}{|c|}{ CMOS } & $0.13 \mu \mathrm{m}$ & $0.13 \mu \mathrm{m}$ & $0.18 \mu \mathrm{m}$ & $0.13 \mu \mathrm{m}$ & $0.16 \mu \mathrm{m}$ \\
\hline \multicolumn{2}{|c|}{$\mathrm{V}_{\mathrm{DD}}[\mathrm{V}]$} & 1.2 & 1.5 & 1.8 & 2.7 & 1.8 \\
\hline \multicolumn{2}{|c|}{ Freq $[\mathrm{GHz}]$} & 2 & 2.4 & 5.2 & 1.96 & 0.9 \\
\hline \multicolumn{2}{|c|}{ Inductor Number } & 0 & 1 & 2 & 0 & 0 \\
\hline \multicolumn{2}{|c|}{ Flicker NF reduction $[\mathrm{dB}]$} & 7@10kHz & 7.5@10kHz & 7@10kHz & $9.5 @ 10 \mathrm{kHz}$ & $9 @ 1 \mathrm{kHz}$ \\
\hline \multirow{2}{*}{$\begin{array}{c}\text { Flicker NF } \\
@ 10 \mathrm{kHz}[\mathrm{dB}]\end{array}$} & w/o cancelling & 21.8 & 37 & 27 & 18 & 20 \\
\hline & w/ cancelling & 14 & 29 & 20 & 8.5 & 13 \\
\hline \multicolumn{2}{|c|}{$\begin{array}{l}\text { Gain Improvement } \\
{[\mathrm{dB}]}\end{array}$} & 0.5 & 1.3 & 6 & 2 & 5 \\
\hline \multirow[t]{2}{*}{ Gain $[\mathrm{dB}]$} & w/o cancelling & 0 & 10.1 & 9.3 & 5 & 12.3 \\
\hline & w/ cancelling & 0.5 & 11.4 & 16.2 & 7 & 17.6 \\
\hline \multicolumn{2}{|c|}{ Thermal NF changes* $[\mathrm{dB}]$} & 0 & 0 & 0 & -2 & +0.1 \\
\hline \multirow{2}{*}{$\begin{array}{c}\text { Thermal NF } \\
@ 10 \mathrm{MHz}[\mathrm{dB}]\end{array}$} & w/o cancelling & 12 & $7.5^{\#}$ & 10 & 8.5 & 10 \\
\hline & w/ cancelling & 12 & $7.5^{\#}$ & 10 & 6.5 & 10.1 \\
\hline \multicolumn{2}{|c|}{ IIP3 improvement [dB] } & 0 & 1.6 & 0 & 1 & 10 \\
\hline \multirow[b]{2}{*}{ IIP3 [dBm] } & w/o cancelling & 10.5 & 3.8 & -4 & 1 & 1.8 \\
\hline & w/ cancelling & 10.5 & 4.4 & -5 & 2 & 11.8 \\
\hline \multicolumn{2}{|c|}{ Bias current increases } & $0 \%$ & $0 \%$ & $0 \%$ & N/A & $16 \%$ \\
\hline \multirow{2}{*}{$\begin{array}{c}\text { Bias current } \\
{[\mathrm{mA}]}\end{array}$} & w/o cancelling & 2 & 6 & 3.9 & 3 & 9.2 \\
\hline & w/ cancelling & 2 & 6 & 3.9 & 3 & 10.9 \\
\hline
\end{tabular}

* “+” for NF increases and “-" for NF decreases. 